\title{
Optical and electronic properties of colloidal CdSe Quantum Rings
}

${ }^{1}$ James Xiao, ${ }^{1}$ Yun Liu, ${ }^{2}$ Violette Steinmetz, ${ }^{1}$ Mustafa Caglar, ${ }^{1}$ Jeffrey Mc Hugh, ${ }^{1}$ Tomi Baikie, ${ }^{1}$ Nicolas Gauriot, ${ }^{1}$ Malgorzata Nguyen, ${ }^{1}$ Eduardo Ruggeri, ${ }^{1}$ Zahra Andaji-Garmaroudi, ${ }^{1}$ Samuel D. Stranks, ${ }^{2}$ Laurent Legrand, ${ }^{2}$ Thierry Barisien, ${ }^{1}$ Richard H. Friend, ${ }^{1}$ Neil C. Greenham, ${ }^{1}$ Akshay Rao* and ${ }^{1}$ Raj Pandya*

*correspondence: ar525@cam.ac.uk,rp558@cam.ac.uk

${ }^{1}$ Cavendish Laboratory, University of Cambridge, J.J. Thomson Avenue, CB3 0HE, Cambridge, United Kingdom

${ }^{2}$ Sorbonne Université CNRS-UMR 7588, Institut des NanoSciences de Paris, INSP, 4 place Jussieu, F75005 Paris, France

\section{Supporting Information}

Contents

S1: Transmission Electron Microscopy Images of Partially Etched nanoplatelets 2

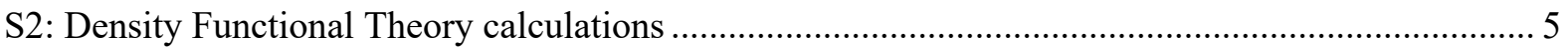

S3: Temperature dependent X-Ray diffraction and Single nanoring stability and PL histograms ......... 6

S4: Dynamic light scattering and zeta-potential measurements ...................................................... 9

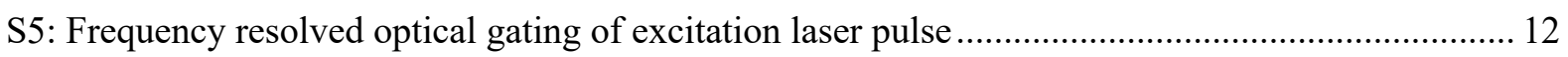

S6: Pump energy dependence of transient absorption dynamics ...................................................... 13

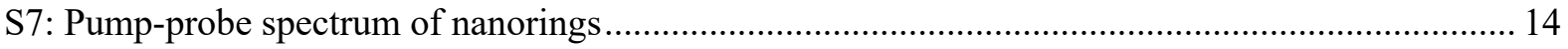

S8: Extraction of mode frequencies from impulsive vibrational spectrum ........................................ 17

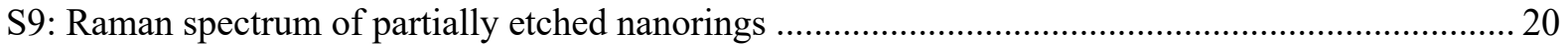

S10: Temperature dependent absorption and emission of nanoplatelets and nanorings....................... 21

S11: Cryogenic transient photoluminescence spectra of nanoplatelets and nanorings ......................... 23

S12: Modelling surface emission from nanoplatelets and naorings ................................................... 25

S13: Room temperature transient photoluminescence spectra of nanoplatelets and nanorings............ 28

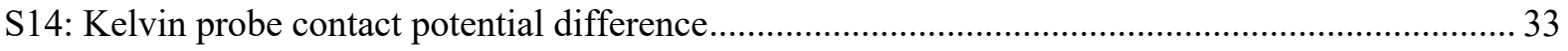

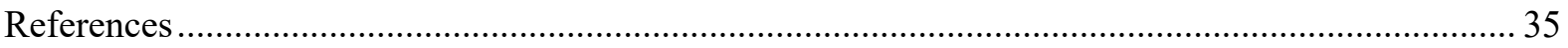


Aliquots of partially etched nanoplatelets, which later developed into fully perforated nanorings, were taken at various time intervals. Transmission electron microscopy image below show that in the case of partially etched nanorings, a thin layer of CdSe remains at the centre of the platelet and/or multiple rings appear to be present within the structure.

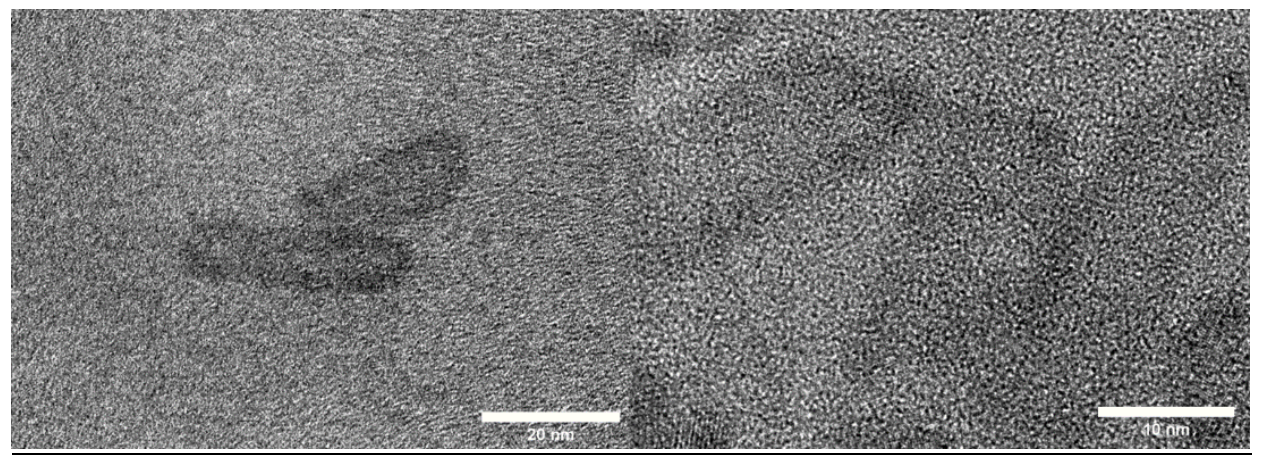

Figure S1: Transmission electron microscopy (TEM) images of partially etched nanoplatelets approximately 3 (left) and 5 (right) mins after addition of etchant.

Figure S1i shows additional TEM images of nanorings and nanoplatelets. The concentration of nanoring and nanoplatelet solution dropcast onto the grids was similar to that used for Kelvin probe measurements. Nanorings tend to lie flat on the TEM grid and sit isolated from one another, whereas nanoplatelets sit flat as well as lying on their edge. Nanoplatelets tend to stack also whereas empirically no nanoring stacks can be observed. This may be as a consequence of their irregular shape which disfavours stacking. 
a

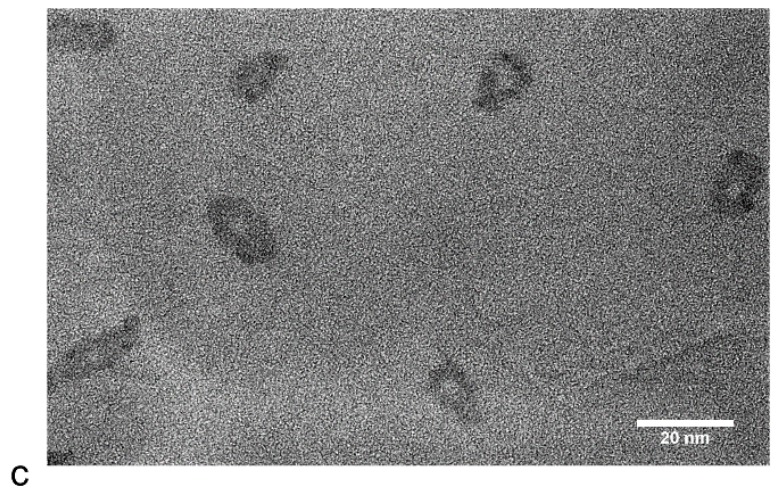

C

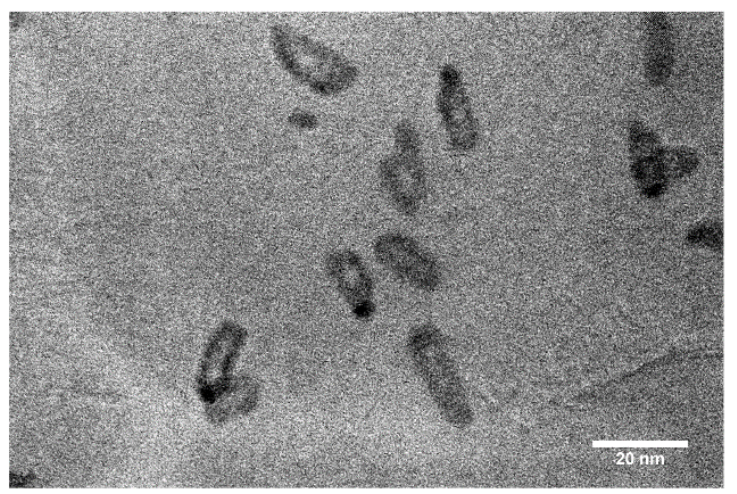

b
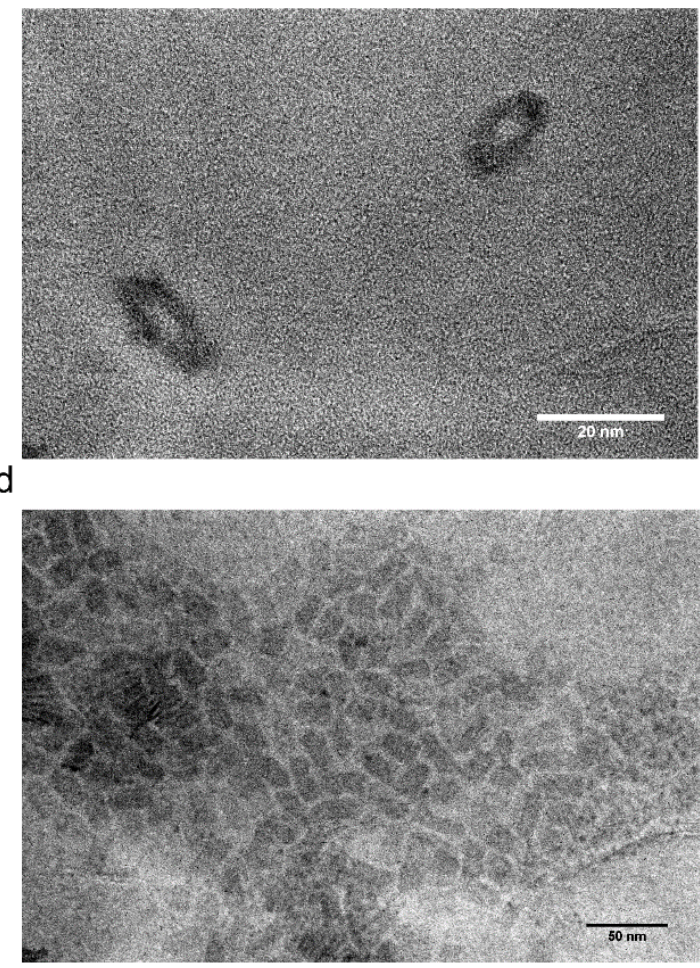

Figure S1i: a-b. Transmission electron microscopy images of nanorings and nanoplatelets. Transmission electron microscopy images of nanorings. Nanorings tend to be relatively isolated from one another and lie flat when drop cast onto the TEM grids. c. When stacking nanorings tend to lie vertically, this may be because of unfavourable stacking interactions between the asymmetrically distributed objects. This TEM image is for partially etched samples towards the end of the etching cycle (10 mins). d. Nanoplatelets on the other hand tend to stack easily and can lie both flat and on their edge.

When the aspect ratio of nanoplatelets is $>1.5$ the ring etch produces a double nanoring structure, which have an absorption edge red shifted as compared to single rings (Figure S1ii)

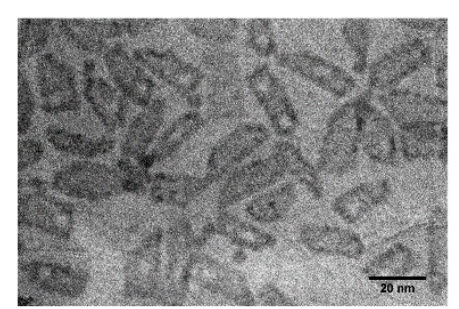

b

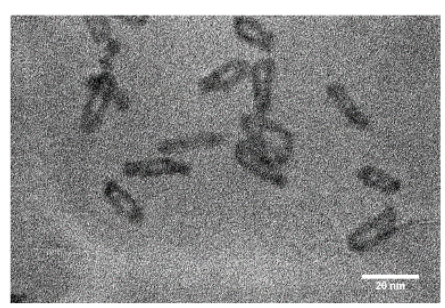

C

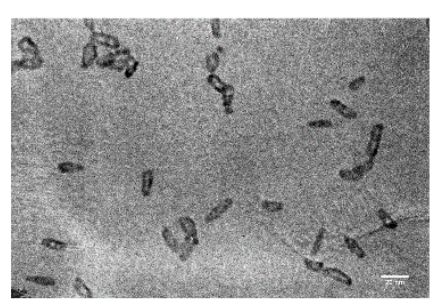

Figure S1ii: a-c. Transmission electron microscopy images of double nanorings. When the aspect ratio (length:width) of nanoplatelets is $>1.5$ it is typically found the rings etch to form two holes. Spectroscopically these double rings have an absorption edge red shifted from the single ring material. 
When nanoplatelet structures with a large size $>15 \mathrm{~nm}$ are used as a precursor for the ring etch, nanowaffle type structures with large number of holes are formed as opposed to a single large nanoring (Figure S1iii).

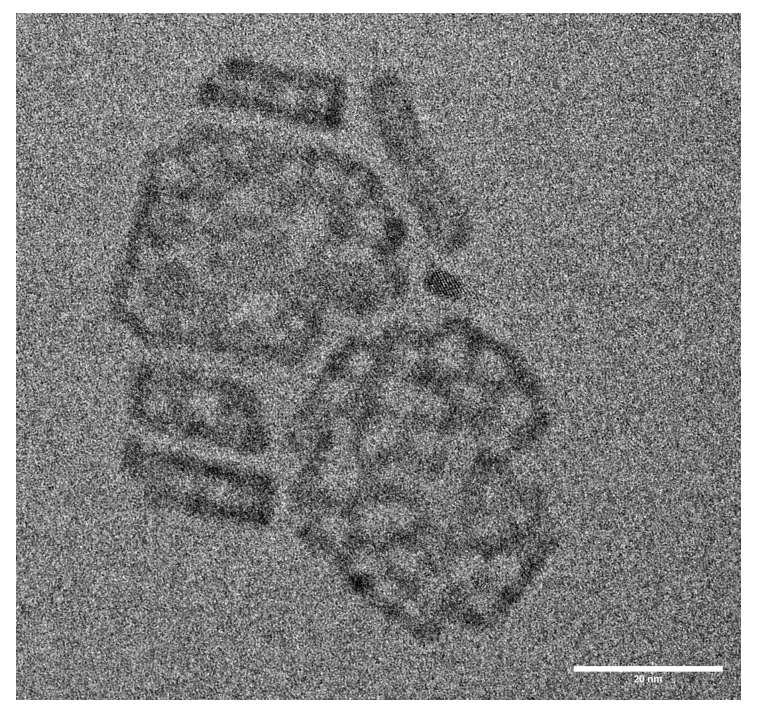

Figure S1iii: Nanowaffle structures generated by etching of low aspect ratio $35 \times 35 \mathrm{~nm}$ nanoplatelets.

Finally, we remark that in attempt to understand the influence of ligands on the properties of nanorings post synthetic treatment with chloride ligands was attempted through using two methods. $\mathrm{CdCl}_{2}$ was dissolved in either methanol or oleylamine $(0.2 \mathrm{~mL}, 50 \mathrm{mM})$ and added to a stirring solution of nanorings $(1 \mathrm{~mL}, 5 \mathrm{mg} / \mathrm{mL})$ in hexane at $60 \mathrm{C}$ and held for 5 minutes. The treated rings were then precipitated by addition of acetone and centrifuged. For both treatment methods, the precipitated QRs did not redisperse in hexane following removal of the anti-solvent. ${ }^{1,2}$ 
S2: Density Functional Theory calculations

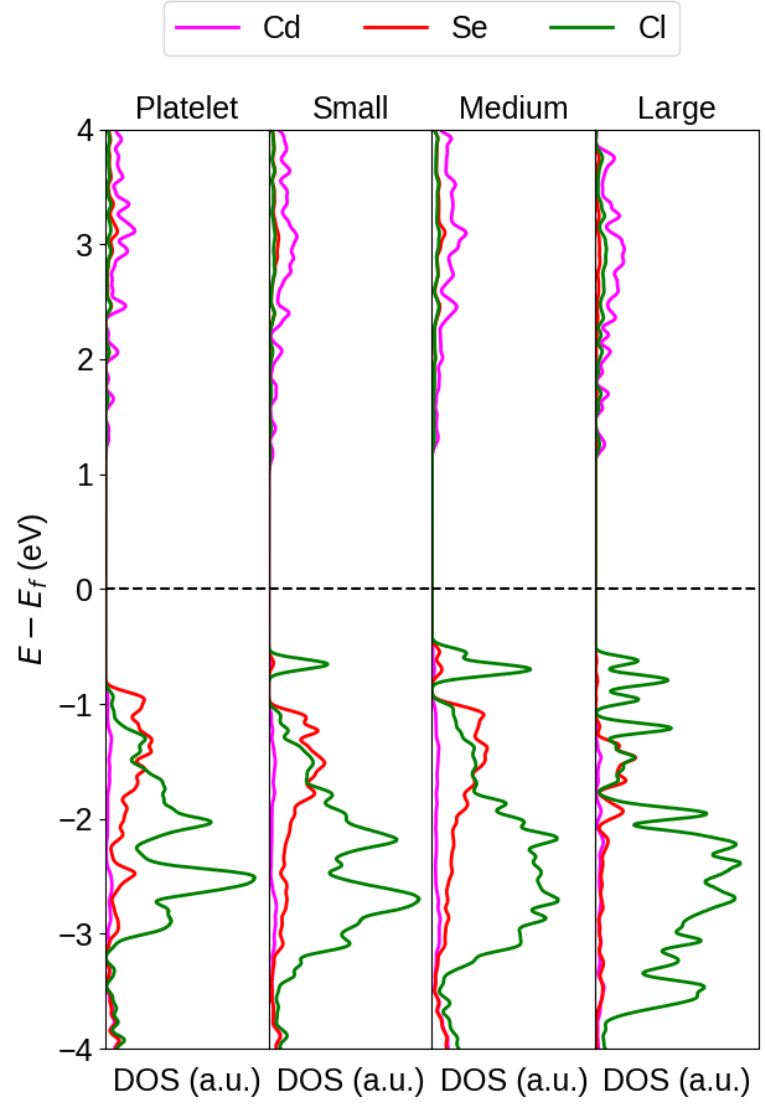

Figure S2: The projected density of states (PDOS) of the nanoplatelet, small, medium and large nanorings. The Fermi level is indicated by the horizontal dashed line.

a)

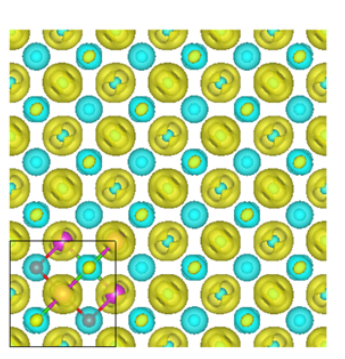

b)

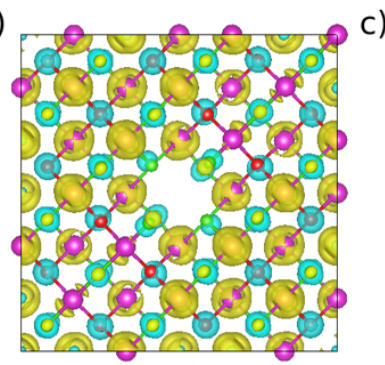

c)

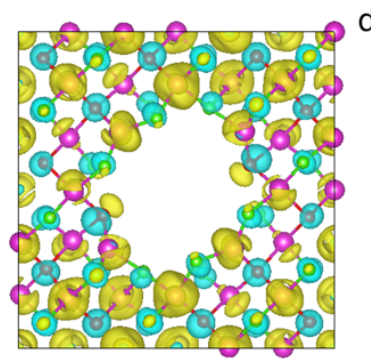

d)

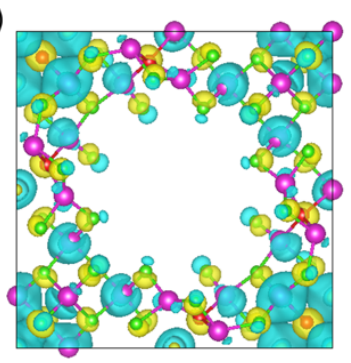

Figure S2i: a. The top view of the ball-and-stick model of the a $3 \times 3$ supercell, with the unit cell indicated by the black solid line. The $\mathrm{Cd}, \mathrm{Se}$ and $\mathrm{Cl}$ atoms are represented by magenta, red and green spheres, and the isosurface of the CBM wavefunction are in cyan and yellow. b-d The CBM wavefunction of the nanorings of small, medium and large sizes respectively. 
S3: Temperature dependent X-Ray diffraction and Single nanoring stability and PL histograms

Temperature dependent X-Ray diffraction measurements were performed to ensure there was no phase change during the cooling of both nanoplatelets and nanorings. As can be seen from the plots below no discontinuity in the X-Ray diffraction patter is observed, with very little change in peak positions, consistent with minimal changes in the lattice structure. ${ }^{3}$

a

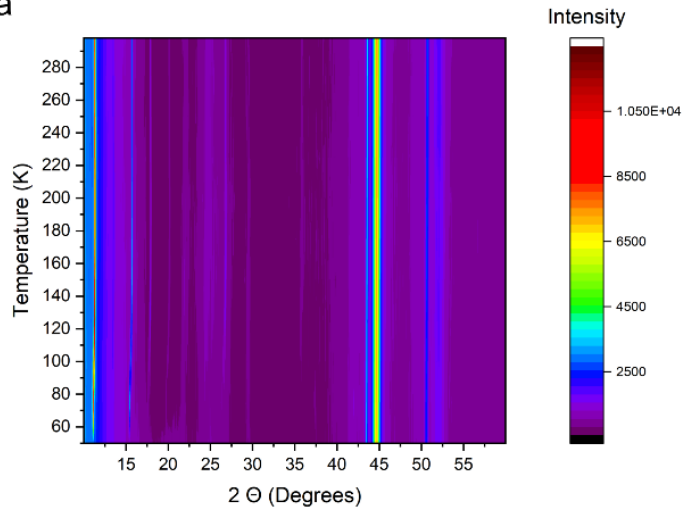

b

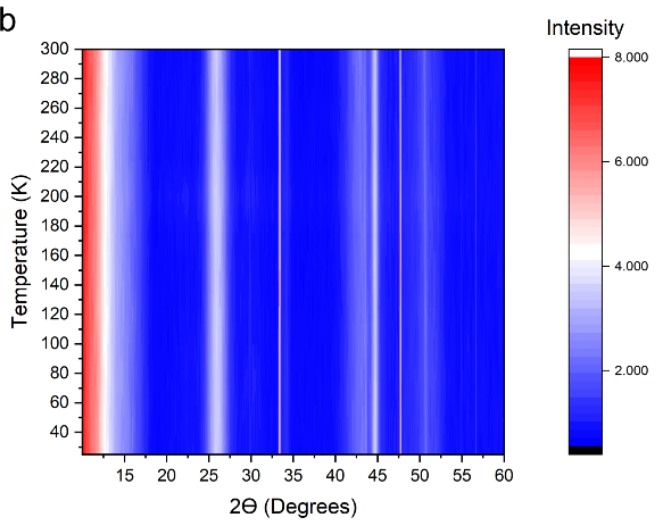

Figure S3: a-b. Temperature dependent X-Ray diffraction pattern of nanoplatelets (left) and nanorings (right). The peaks are at $26^{\circ}, 44^{\circ}$ and $51^{\circ}$ are assigned to the (111), (220) and (311) lattice planes of CdSe respectively and do not shift their positions significantly with temperature. The remaining peaks are assigned to the Si substrate and heating stage.

The PL intensity of single nanorings rapidly diminishes even under low excitation powers $\left(<1 \mu \mathrm{W} / \mu \mathrm{m}^{2}\right)$ as shown in Figure S3i, this could be due to photocharging or sample degradation. This precluded more in-depth single particle measurements, e.g. blinking or polarisation resolved studies and hence future efforts should be directed at increasing the stability of such materials.

Figure S3ii shows a histogram of 14 single nanoring PL energies at $4 \mathrm{~K}$ overlay with the ensemble PL spectrum at $4 \mathrm{~K}$. The histogram matches well the ensemble spectrum suggesting inhomogeneous broadening is the dominant mechanism of PL broadening in nanorings. 


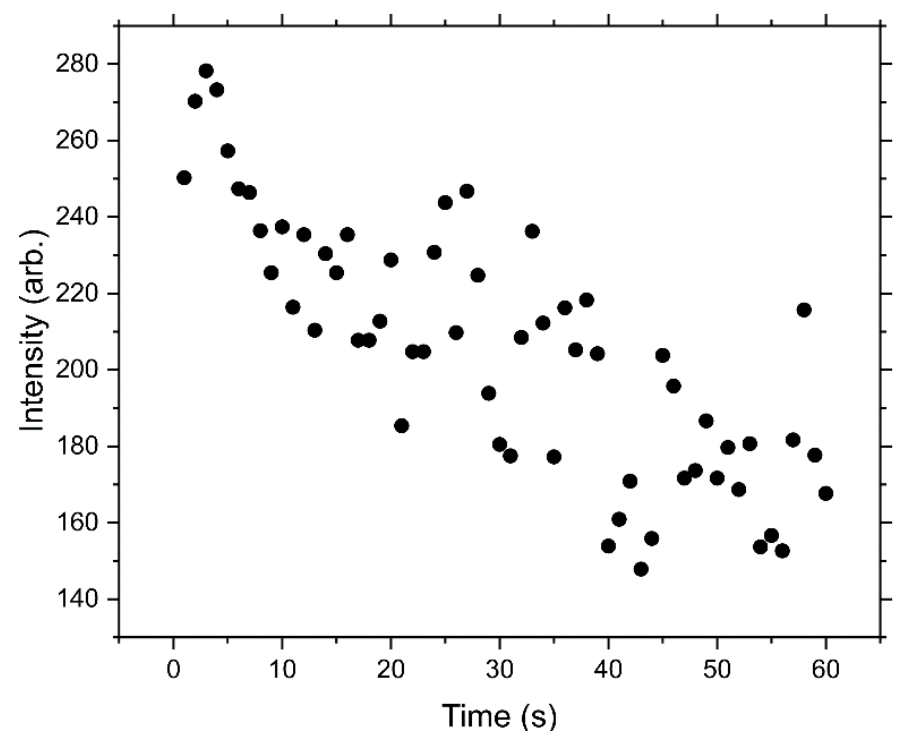

Figure S3i: PL intensity for single nanoring over 60 s. The PL intensity drops by $50 \%$ in this time period, reflecting the low PL stability.

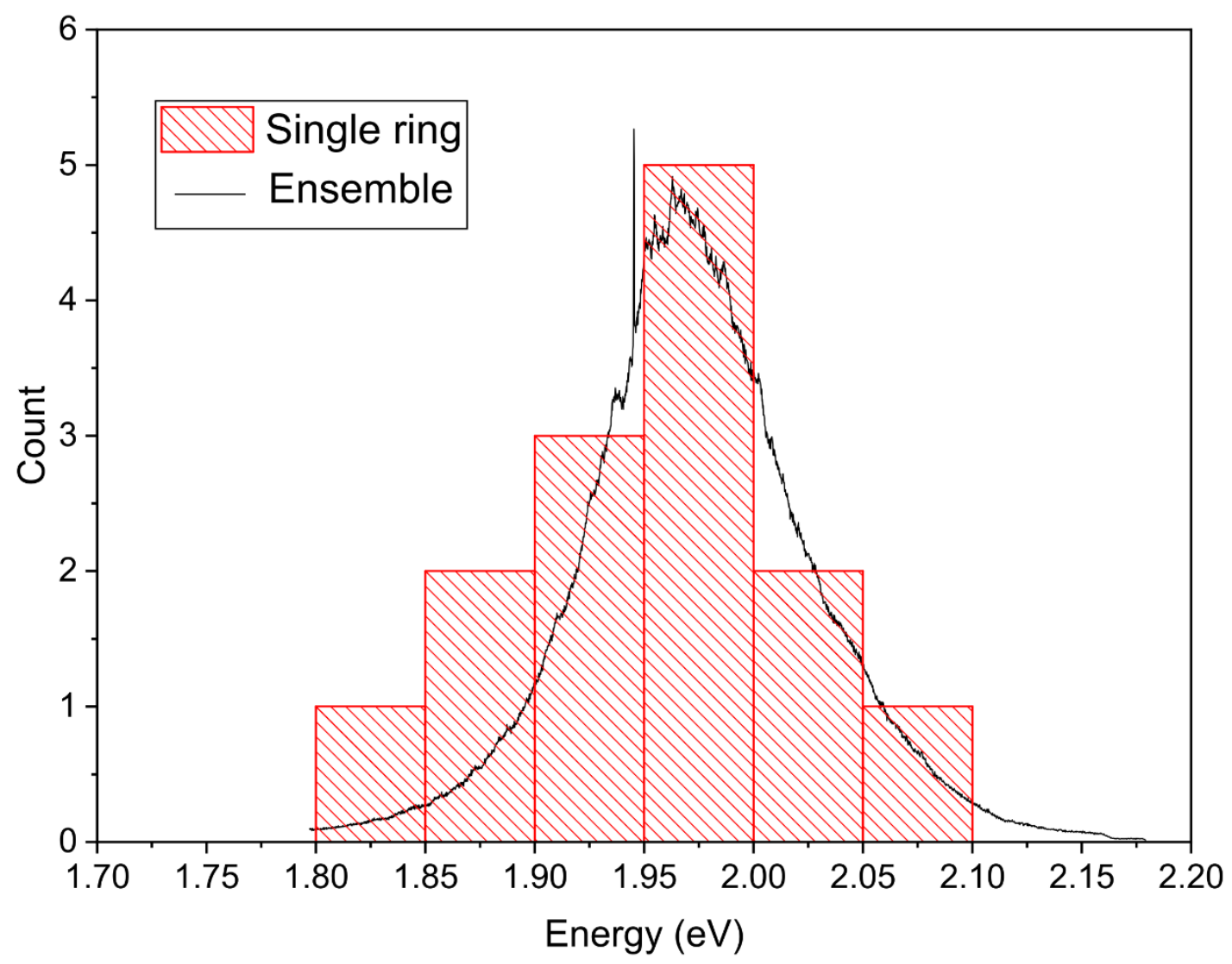

Figure SI3ii: Histogram (red) of single nanoring energies for 14 nanorings overlay with the ensemble PL spectrum (black). Despite the small sample size, the histogram well matches the ensemble spectrum 
suggesting inhomogeneous broadening is the dominant contributor to the broad room temperature linewidth. 
S4: Dynamic light scattering and zeta-potential measurements

Figure SI4 and SI4i show dynamic light scattering measurements of nanorings and nanoplatelets to qualitatively assess their size and distribution in solution. Intensity distributions of particle size were derived by fitting the measured intensity autocorrelation function with a non-negatively constrained least squares (NNLS) fit, considering the intensity autocorrelation to be composed of 70 individual decays. Number distributions as shown in Figure S4 and S4i were generated from the derived intensity distributions assuming spherical particles, a refractive index of the solvent (hexane) of $n_{\text {solvent }}=1.375$, and a refractive index of the QDs of $n_{\mathrm{QD}}=2.64 .{ }^{4}$ Size distributions were averaged over 3 runs, each of which consisted of 13-16 individual measurements. The range of size distributions is established by looking at $2 \times$ standard deviation from the mean $(2 \sigma)$.

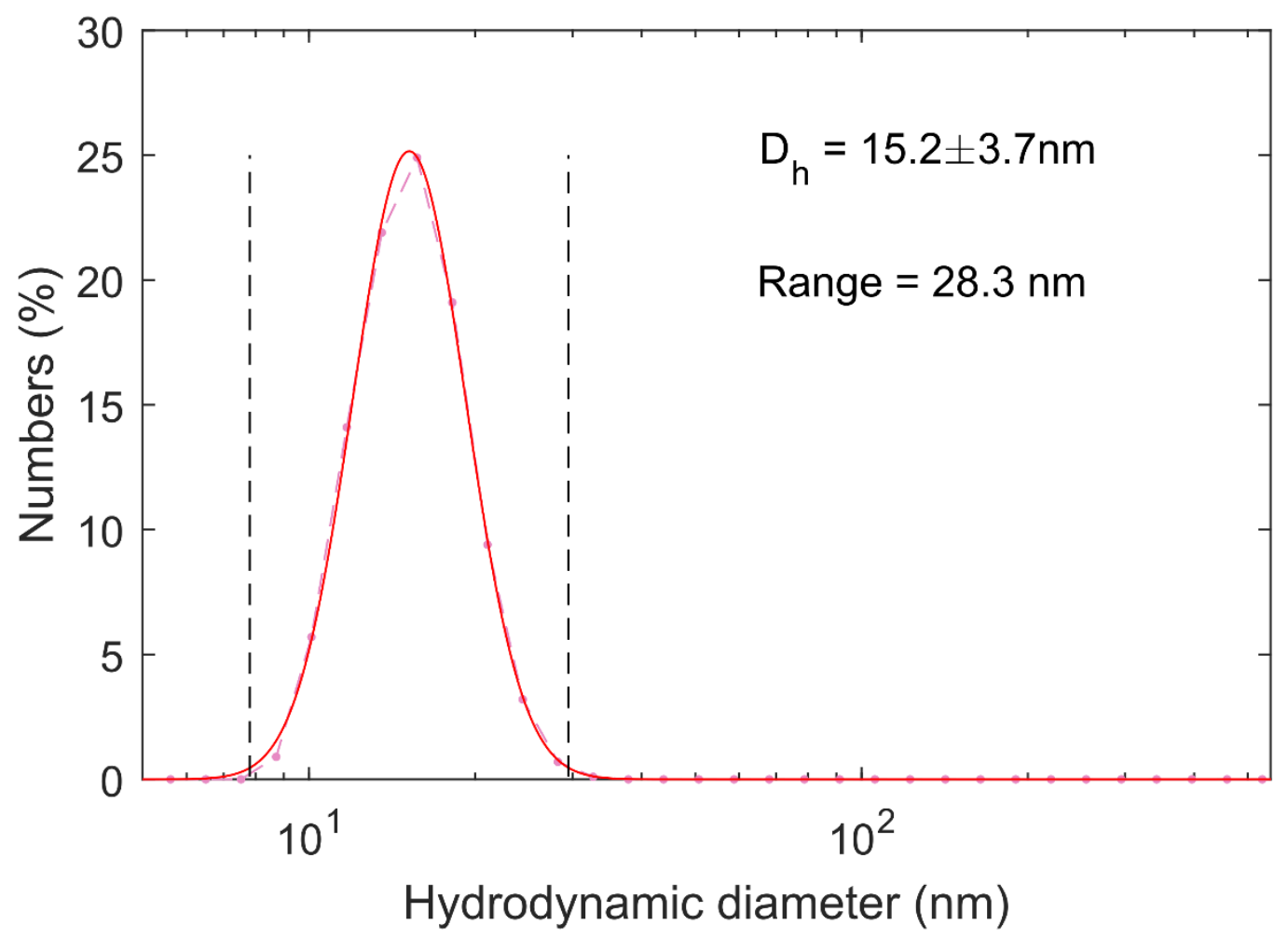

Figure S4: Dynamic light scattering (DLS) measurements of nanorings, showing a mean particle size of $15.2 \pm 3.7 \mathrm{~nm}$. The range of particle sizes is $28.3 \mathrm{~nm}$. 


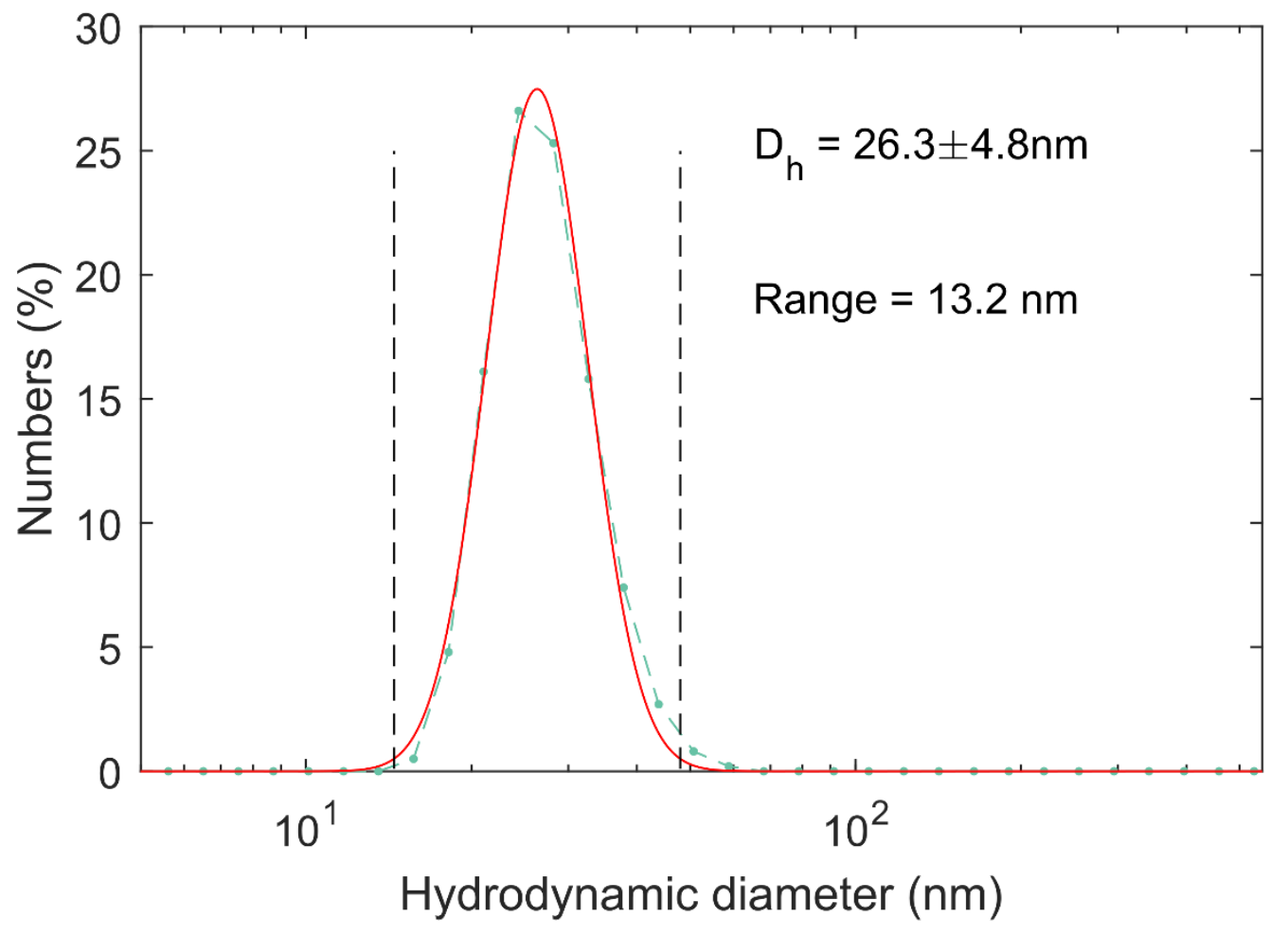

Figure S4i: Dynamic light scattering (DLS) measurements of nanoplatelets, showing a mean particle size of $26.3 \pm 4.8 \mathrm{~nm}$. The range of particle sizes is $13.2 \mathrm{~nm}$. 
Zeta-potential measurements were performed using a commercial Zetasizer with nanoplatelets and nanorings diluted in toluene. Where nanoplatelets have a small positive surface charge of $\sim 10 \mathrm{mV}$, similar to that observed in previous studies, ${ }^{5}$ the charge on nanorings is strongly negative $\sim-50 \mathrm{mV}$, suggestive of a unpassivated selenium rich surface.

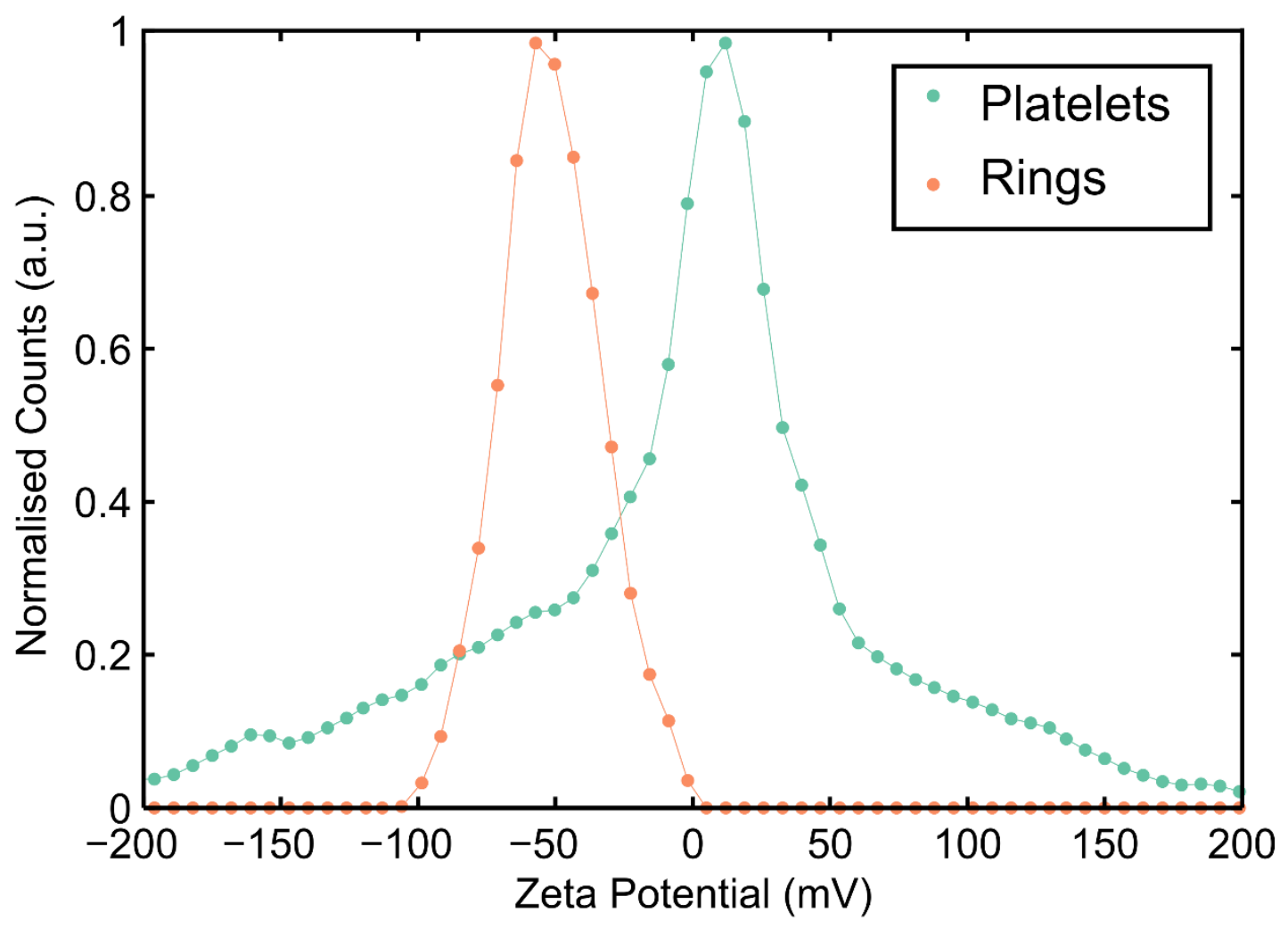

Figure S4ii: Zeta potential measurements of nanoplatelets (green) and nanorings (orange). The nanoplatelets are weakly charged $\sim 10 \mathrm{mV}$, whereas rings have a string negative chare $\sim-50 \mathrm{mV}$. 
S5: Frequency resolved optical gating of excitation laser pulse

The temporal profile of the exciting laser pulse was assessed using frequency resolved optical gating (FROG) $)^{6}$, with second and third order dispersion controlled using a combination of chirp mirrors and wedge prisms. As can be seen from the spectrum below a pulse centred $\sim 500 \mathrm{~nm}, \sim 10 \mathrm{fs}$ in temporal duration can be achieved.

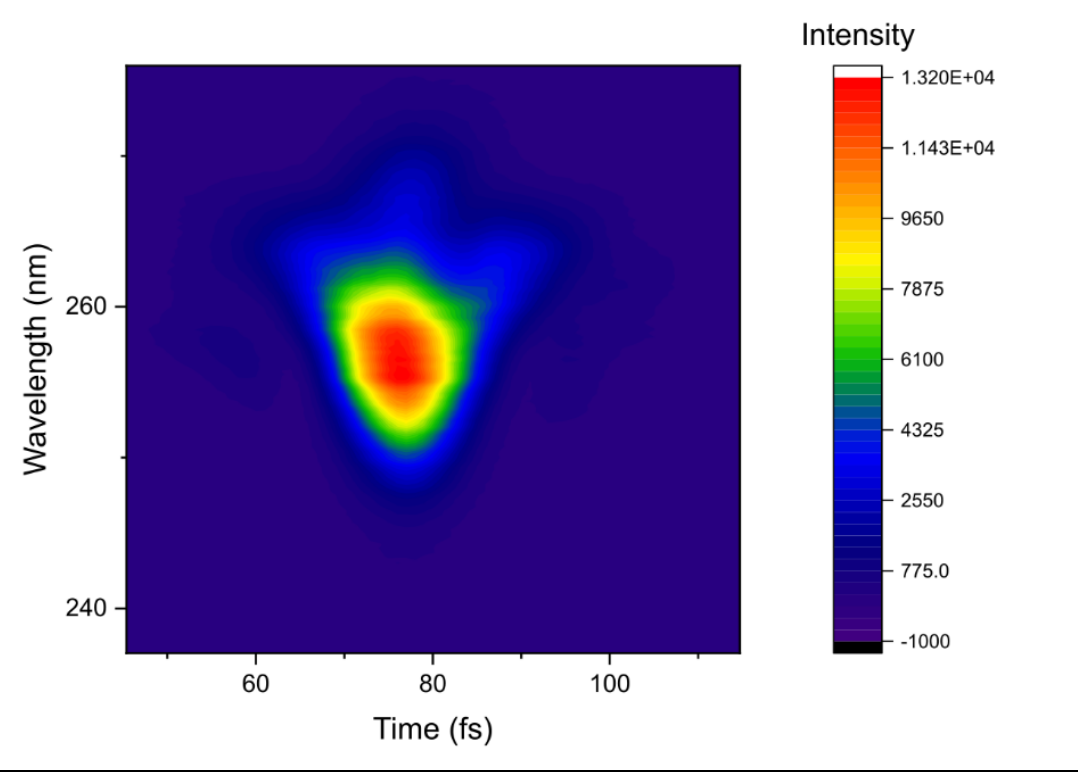

Figure S5: Frequency resolved optical gating (FROG) spectrum of exciting laser pulse used in main text of manuscript centred $\sim 500 \mathrm{~nm}$. 
S6: Pump energy dependence of transient absorption dynamics

a

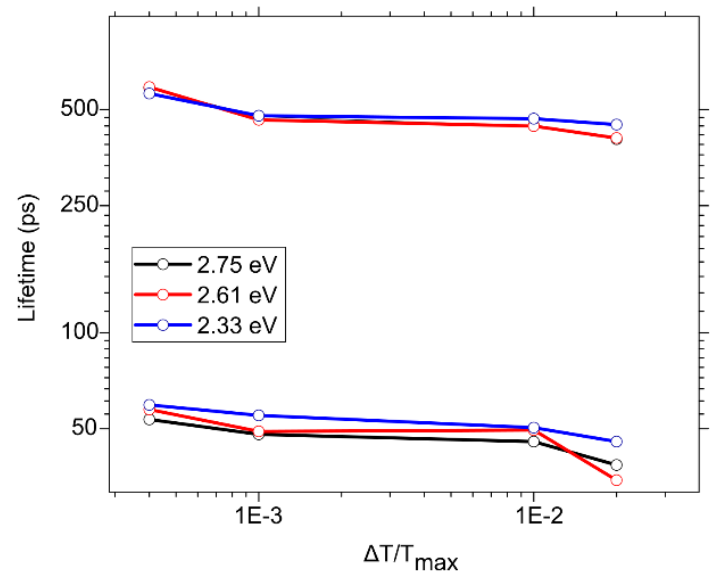

b

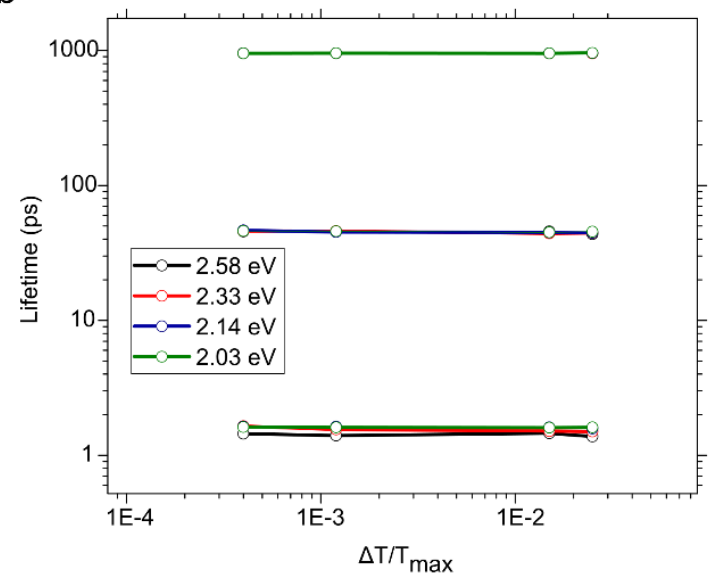

Figure S6: Pump energy and intensity dependence of transient absorption dynamics in nanoplatelets and nanorings. a. In both nanoplatelets and nanorings the ground state bleach shows a multi-exponential decay. For nanoplatelets pumping at higher energies away from the band edge and with higher pump intensities results in faster decay components. This is suggestive of cooling and Auger recombination within a band like picture of excitations. b. In nanorings there is no pump energy or intensity dependence in the dynamics (in the excitation regime studied here where there is less than 1 excitation per particle; see $\mathbf{S 7}$ for fluence dependence and carrier concentration calculations). In both panels the error on the fitted decay is sufficiently small $( \pm 10 \mathrm{ps})$ that is not shown so as to maintain clarity.

Using a narrow-band excitation pulse (FWHM $\sim 10 \mathrm{~nm}$, pulse duration $\sim 200 \mathrm{fs}$ ), we can also study the excitation energy and laser intensity dependence of transitions in both rings and platelets (Figure S6; we note that for each measurement a similar carrier concentration is maintained between nanoplatelets and nanorings (see S7)). In the case of nanoplatelets on increasing both the pump fluence and energy there is a qualitative shortening of the GSB lifetime (Figure S6a). This is in keeping with a model of state filling within a valence band where the cooling time decreases when pumping closer to the band edge and Auger recombination increases with greater excitation densities. ${ }^{7,8}$ In contrast, nanorings do not appear to show any pump energy or excitation density dependence in their transient absorption response. This would again fit with a trap-like picture where excitons hop from site-to-site before localizing, likely assisted by the $200 \mathrm{~cm}^{-1}$ phonon mode, as opposed to being delocalized in band states. Regardless of pump energy and intensity, in both nanorings and nanoplatelets cooling times remain on the sub-picosecond timescale, suggesting as in 0D CdSe QDs the phonon bottleneck is bypassed e.g. by transfer of energy from hot electrons to holes. ${ }^{9}$ In nanorings, our results suggest that any excess energy is also rapidly dissipated before any localization occurs. 
S7: Pump-probe spectrum of nanorings

The kinetics of the decay for both nanoplatelets and nanorings was fit to an equation of the form:

$$
\mathrm{y}=\sum_{\mathrm{n}} \frac{1}{2} \cdot \mathrm{A}_{\mathrm{n}} \cdot \mathrm{e}^{\frac{\mathrm{s}^{2}}{2 \tau_{\mathrm{n}}{ }^{2}}} \cdot \mathrm{e}^{\frac{-\left(\mathrm{t}-\mathrm{t}_{0}\right)}{\tau_{\mathrm{n}}}} \cdot\left(1+\operatorname{erf}\left(\frac{\mathrm{t}-\mathrm{t}_{0}-\frac{\mathrm{s}^{2}}{\tau_{\mathrm{n}}}}{\sqrt{2} \mathrm{~s}}\right)\right) \quad \text { (Equation E1) }
$$

where $\mathrm{s}$ is the time resolution of the experiment, $\mathrm{t}_{0}$ the zero pump-probe delay time, $\tau_{\mathrm{n}}$ the $\mathrm{n}^{\text {th }}$ decay time constant and $A_{n}$ the amplitude of the $n^{\text {th }}$ decay constant. The various time constants detailed in the main text were then obtained with a typical fit shown below in Figure S7.
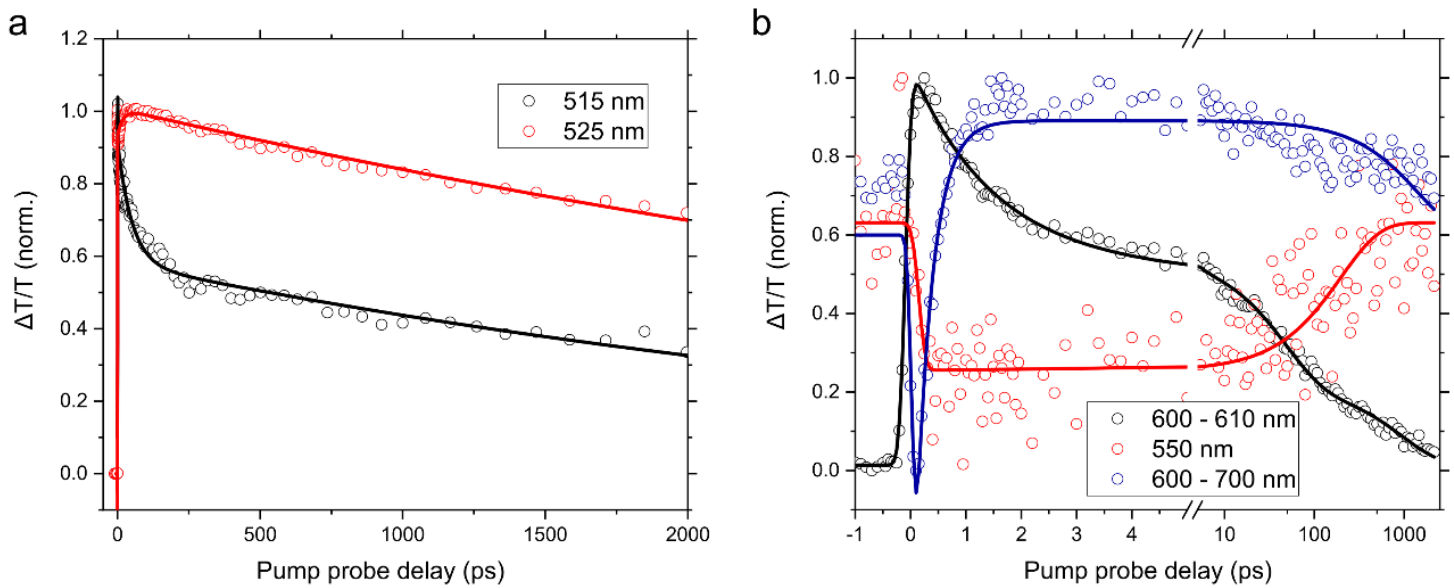

Figure S7: a. Normalized pump-probe decay of GSB feature (red) and band gap renormalization feature (black) in nanoplatelets, fit with equation E1. b. Normalized pump-probe decay of nanorings along GSB (black), renormalization feature (red) and potential sub-gap/defect states (blue).

As discussed in the main text there is a weak pump excitation energy and intensity dependence to the decay of the GSB in nanoplatelets and no observable dependence in nanorings. In the photoluminescence dynamics no pump intensity can be discerned in either nanorings or nanoplatelets. 
a

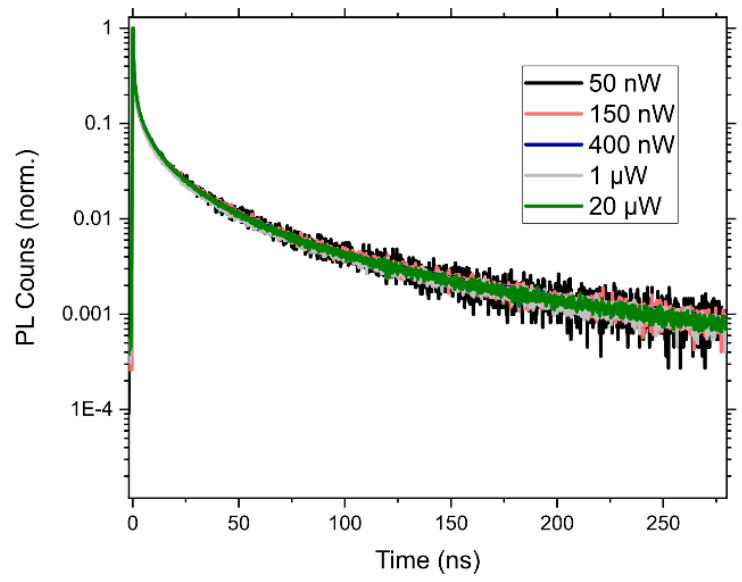

b

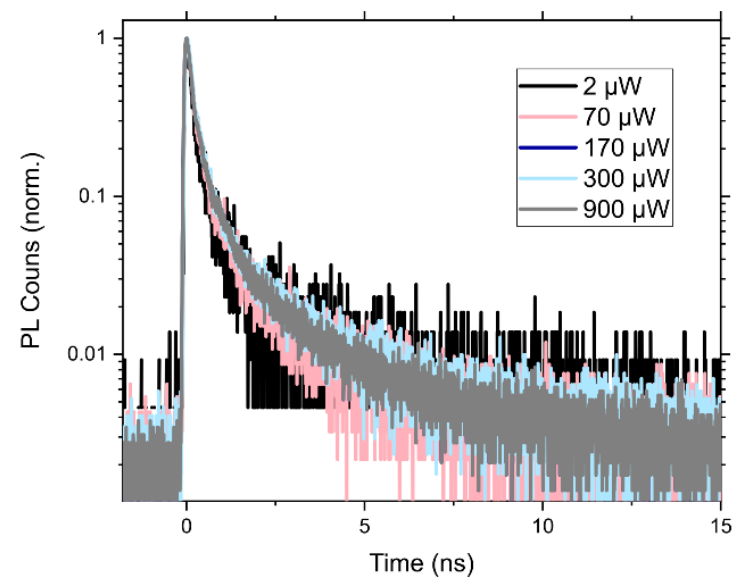

Figure S7i: a-b. Normalized transient photoluminescence decay for nanoplatelets (left) and nanorings (right) in solution. In both cases no strong pump intensity is observed.

To determine whether the red-shift in the pump-probe spectra of nanorings observed over $1 \mathrm{ps}$ originates from energy transfer to rings of a lower band-gap, measurements were repeated in dilute solution $(0.01 \mathrm{mg} / \mathrm{ml}$ in hexane; concentrated $-10 \mathrm{mg} / \mathrm{mL}$ hexane $)$. As shown in Figure S7ii there is a shift of $35 \mathrm{meV}$ in the dilute solution as compared to $50 \mathrm{meV}$ in concentrated solutions of nanorings, suggesting intra-ring FRET to be a small effect in these materials. We can estimate the amount of energy transfer we would expect at $1 \mathrm{ps}$ from the absorption and emission spectra and the quantum yield of nanorings.
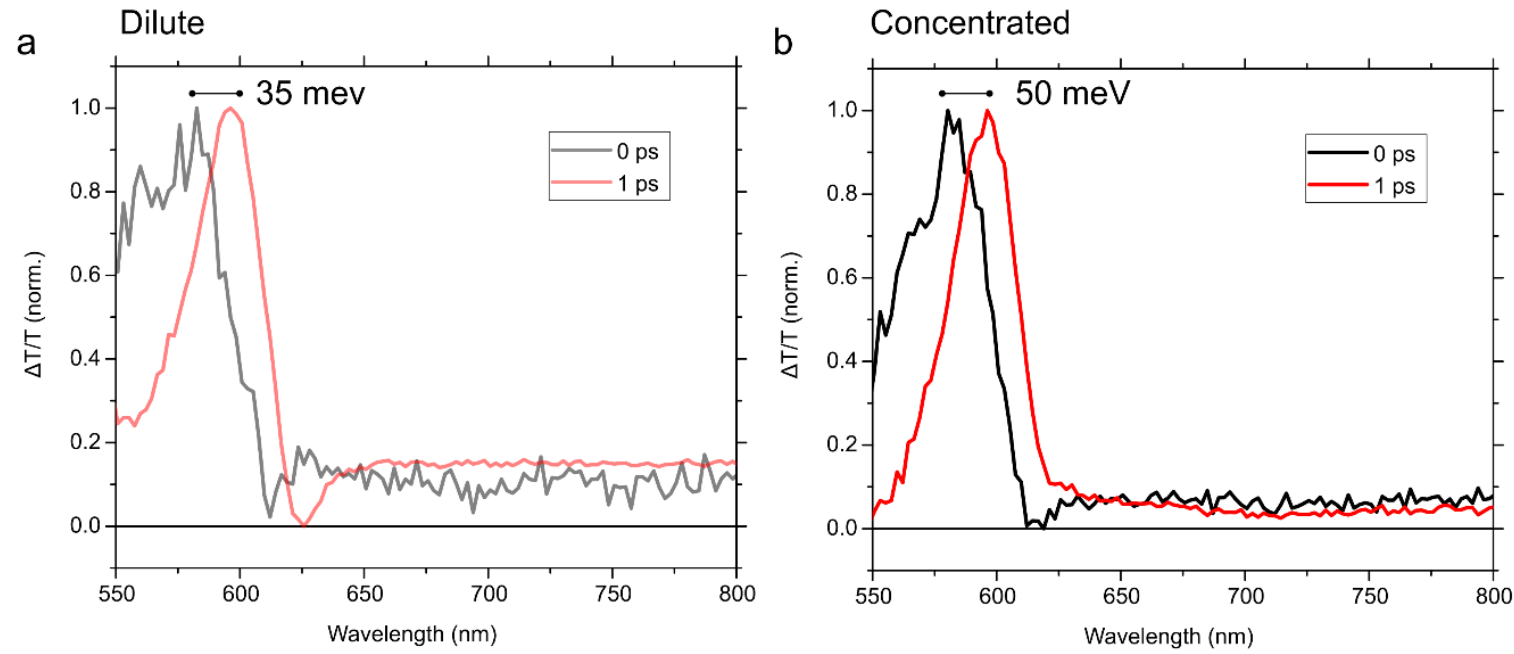

S7ii: a-b Normalised pump-probe spectra of nanorings in dilute and concentrated solution at 0 ps and 1 ps. 
The FRET efficiency between nanorings in concentrated solution can be estimated using the following equation:

$$
\begin{gathered}
E=\frac{1}{1+\left(\frac{r}{R_{0}^{6}}\right)} \text { (Equation E2) } \\
R_{0}^{6}=\frac{2.07}{128 \pi N_{A}} \frac{\kappa Q_{D}}{n^{4}} \int F_{D}(\lambda) \varepsilon_{A}(\lambda) \lambda^{4} d \lambda \text { (Equation E3) }
\end{gathered}
$$

Here $E$ is the FRET efficiency, $r$ the average distance between nanorings in solution (estimated to $0.4 \mathrm{~nm}$ in $10 \mathrm{mg} / \mathrm{mL}$ hexane) and $R_{0}$ the FRET radius. This latter quantity is determined from the overlap of the absorption and emission spectra $F_{D}$, the quantum yield $Q_{D}, n$ the refractive index and an orientation factor $\kappa^{2}$ which is taken as 0.66 here. Inserting the relevant quantities gives a homo-FRET efficiency of $4.36 \%$ for nanorings in $10 \mathrm{mg} / \mathrm{mL}$ hexane suggesting this to be a small effect, in-keeping with the low quantum yield and large Stokes shift of nanorings.

The carrier concentration for nanoplatelets and nanorings used in the experiments described can be determined as: $\zeta=\varphi \times O D$ where $\varphi$ is the photon flux (density of photons per pulse per unit area) and $O D$ which is the absorption cross section of nanoplatelets/nanorings.

\begin{tabular}{|l|l|l|l|l|l|}
\hline $\begin{array}{l}\Delta \mathrm{T} / \mathrm{T} \\
\text { Nanoplatelets }\end{array}$ & $\begin{array}{l}\zeta \\
\text { Nanoplatelets } \\
\left(\times 10^{14}\right. \\
\text { photons } / \mathrm{cm}^{2} \\
\text { per pulse })\end{array}$ & $\begin{array}{l}\# \text { excitations } \\
\text { per object }\end{array}$ & $\begin{array}{l}\Delta \mathrm{T} / \mathrm{T} \\
\text { Nanorings }\end{array}$ & $\begin{array}{l}\zeta \text { Nanorings } \\
\left(\times 10^{14}\right. \\
\text { photons } / \mathrm{cm}^{2} \\
\text { per pulse })\end{array}$ & $\begin{array}{l}\# \text { excitations } \\
\text { per object }\end{array}$ \\
\hline $0.411 \times 10^{-3}$ & 0.129 & 0.01 & $0.472 \times 10^{-3}$ & 0.28 & 0.008 \\
\hline $1.03 \times 10^{-3}$ & 0.231 & 0.02 & $1.19 \times 10^{-3}$ & 0.619 & 0.013 \\
\hline $0.981 \times 10^{-2}$ & 1.93 & 0.30 & $1.46 \times 10^{-2}$ & 2.03 & 0.39 \\
\hline $2.06 \times 10^{-2}$ & 4.10 & 0.73 & $2.39 \times 10^{-2}$ & 4.28 & 0.67 \\
\hline
\end{tabular}

Table T1: Summary of $\Delta \mathrm{T} / \mathrm{T}$ signal strength (at $\mathrm{t}_{0}$ ) and corresponding excitation densities for pumpprobe experiments on nanoplatelets and nanorings used in this work. 
S8: Extraction of mode frequencies from impulsive vibrational spectrum

The pump-probe kinetics for nanoplatelets and nanorings can be fit with an exponential decay. Following fitting and subtraction of the electronic kinetic, the residual is Fourier transformed to give the impulsive Raman spectrum. In the case of nanoplatelets subtracting the electronic decay leaves a residual of noise where no vibronic coupling is observed. However, in the case of nanorings a strong oscillatory component can be observed atop of the electronic decay. This vibronic coupling is only observed around the GSB peak in the transient absorption spectrum of nanorings suggesting this LOphonon mode couples to the ground state potential energy surface. Based on the pump probe delay range a frequency resolution of $\sim 16 \mathrm{~cm}^{-1}$ is achieved. The dephasing time of the mode can be obtained by fitting the residual in Figure S7d with an exponentially decaying sinusoid of the form: $\sum_{\mathrm{n}} \frac{1}{2} \cdot \mathrm{A}_{\mathrm{n}} \cdot \mathrm{e}^{\frac{\mathrm{s}^{2}}{2 \tau_{\mathrm{n}}{ }^{2}}} \cdot \mathrm{e}^{\frac{-\left(\mathrm{t}-\mathrm{t}_{0}\right)}{\tau_{\mathrm{n}}}} \cdot\left(1+\operatorname{erf}\left(\frac{\mathrm{t}-\mathrm{t}_{0}-\frac{\mathrm{s}^{2}}{\tau_{\mathrm{n}}}}{\sqrt{2} \mathrm{~s}}\right)\right) \cdot \sin (\alpha \mathrm{t}+\beta)$, the terms in this equation are the same as those defined in E1 with $\alpha$ and $\beta$ representing empirical fitting parameters. Fitting with a single exponential decay gives a dephasing time of $834 \pm 10 \mathrm{fs}$ with an $\mathrm{R}^{2}$ value on the fit of 0.91 . Figure $\mathrm{S} 7 \mathrm{i}$ shows the wavelength resolved Fourier transform ma, highlighting the mode predominantly modulates the GSB transition of nanorings. 
a

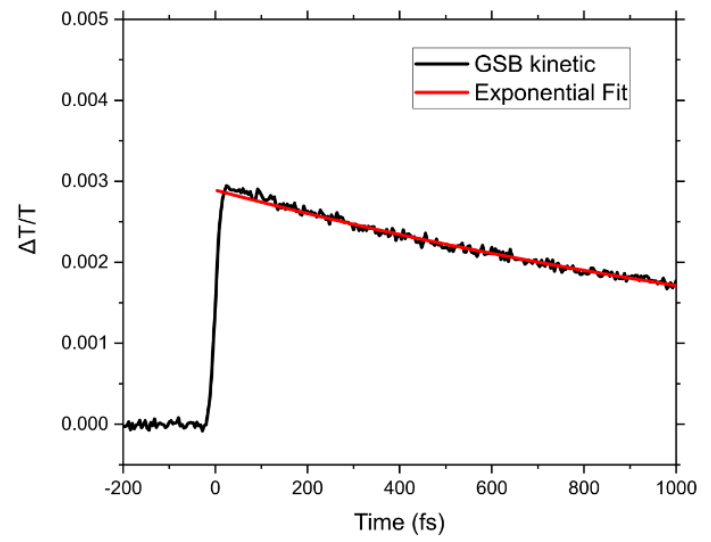

C

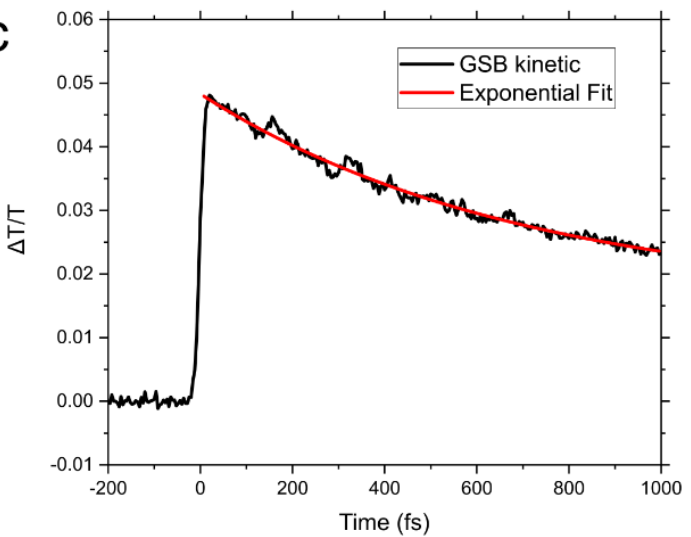

b

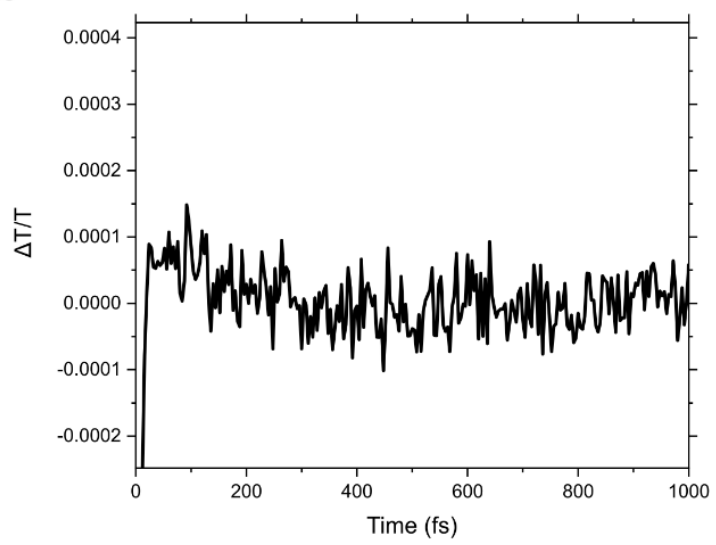

d

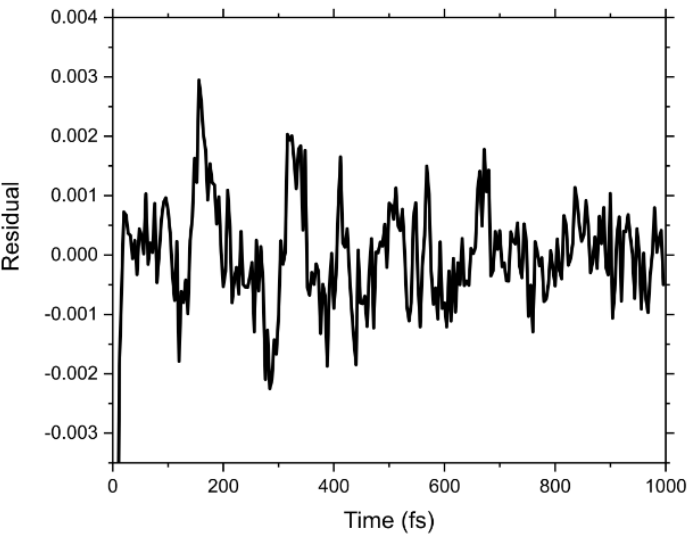

Figure S8: a. Ground state bleach kinetic (GSB) of nanoplatelets (black) with the decay fit with an exponential (red). b. Subtraction of the electronic decay leaves a residual in which no modes can be resolved on Fourier transforming of the spectrum. c. Ground state bleach kinetic (GSB) of nanorings (black) with the decay fit with an exponential (red). d. Clear oscillations can be observed in the residual which when Fourier transformed reveal a CdSe LO-phonon modes at $200 \mathrm{~cm}^{-1}$ (and overtones at 400 $\mathrm{cm}^{-1}$ and $\left.800 \mathrm{~cm}^{-1}\right)$. 


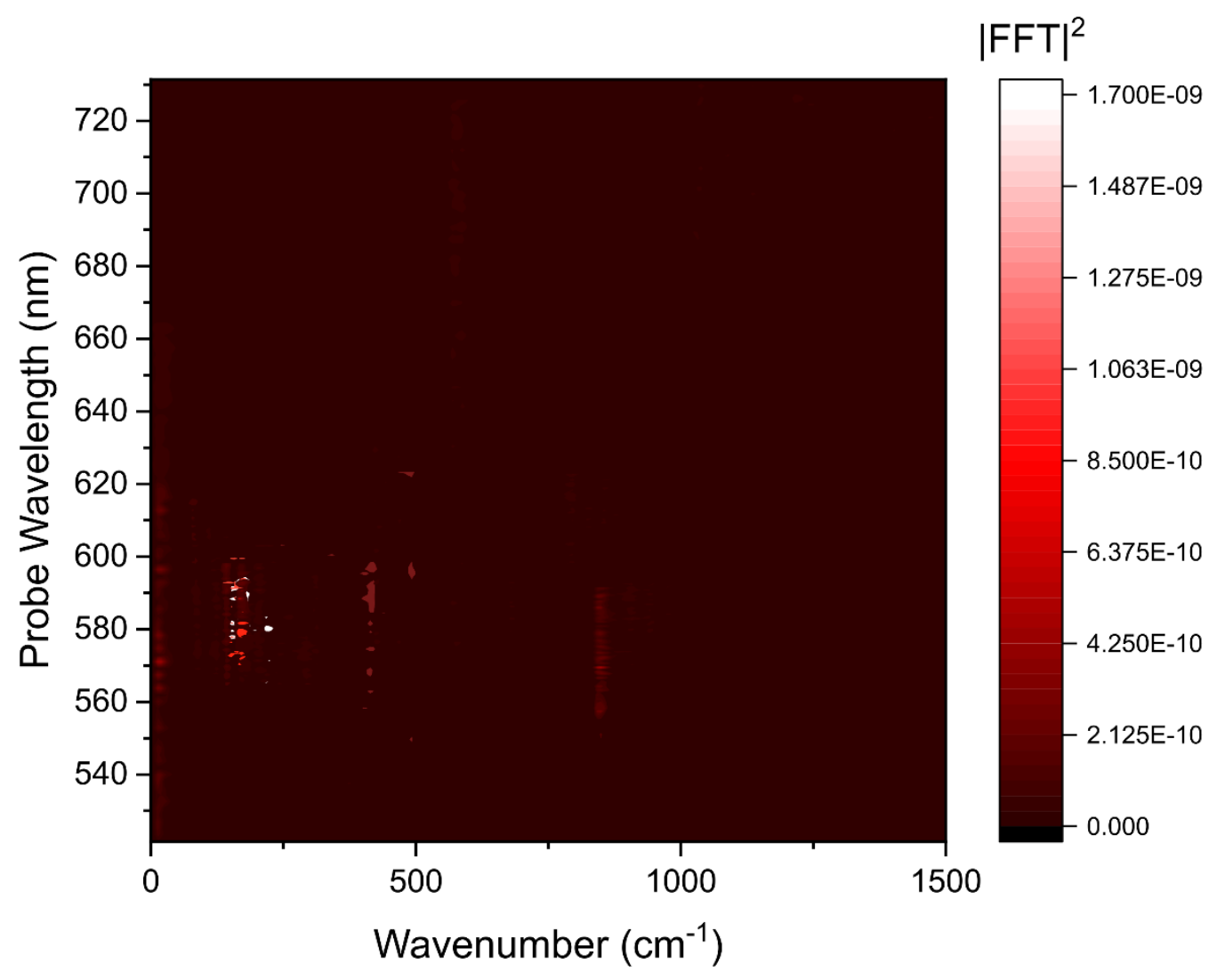

Figure S8i: Wavelength resolved Fourier transform map for nanorings. The CdSe LO-phonon mode at $200 \mathrm{~cm}^{-1}$ (overtones at 400 and $800 \mathrm{~cm}^{-1}$ ) predominantly modulates the GSB transition $560-600 \mathrm{~nm}$.
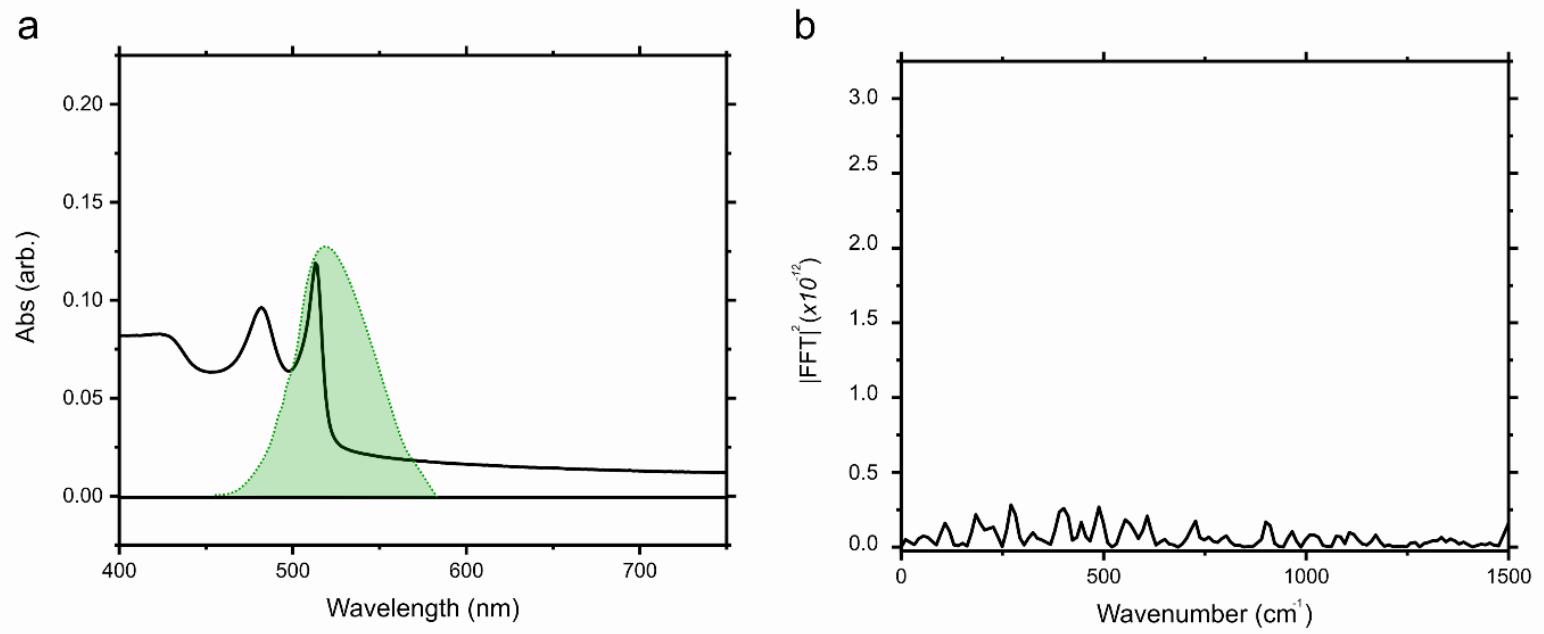

Figure S8ii: Band-edge pumping of the excitonic transitions in nanoplatelets also does not result in the observation of any vibrational modes. This suggests that coupling between the low energy bandedge states is weak ${ }^{10}$. 
S9: Raman spectrum of partially etched nanorings

Aliquots of partially etched nanoplatelets, which later developed into fully perforated nanorings, were taken at various time intervals during the etch process. The samples were dropcast onto glass substrates in an inert $\mathrm{N}_{2}$ environment. Resonant Raman measurements were then performed as detailed in the methods. As can be seen in Figure S9 within 1 min of etching a strong Raman mode at $200 \mathrm{~cm}^{-1}$ appears which has been attributed to CdSe LO-phonons. ${ }^{11}$

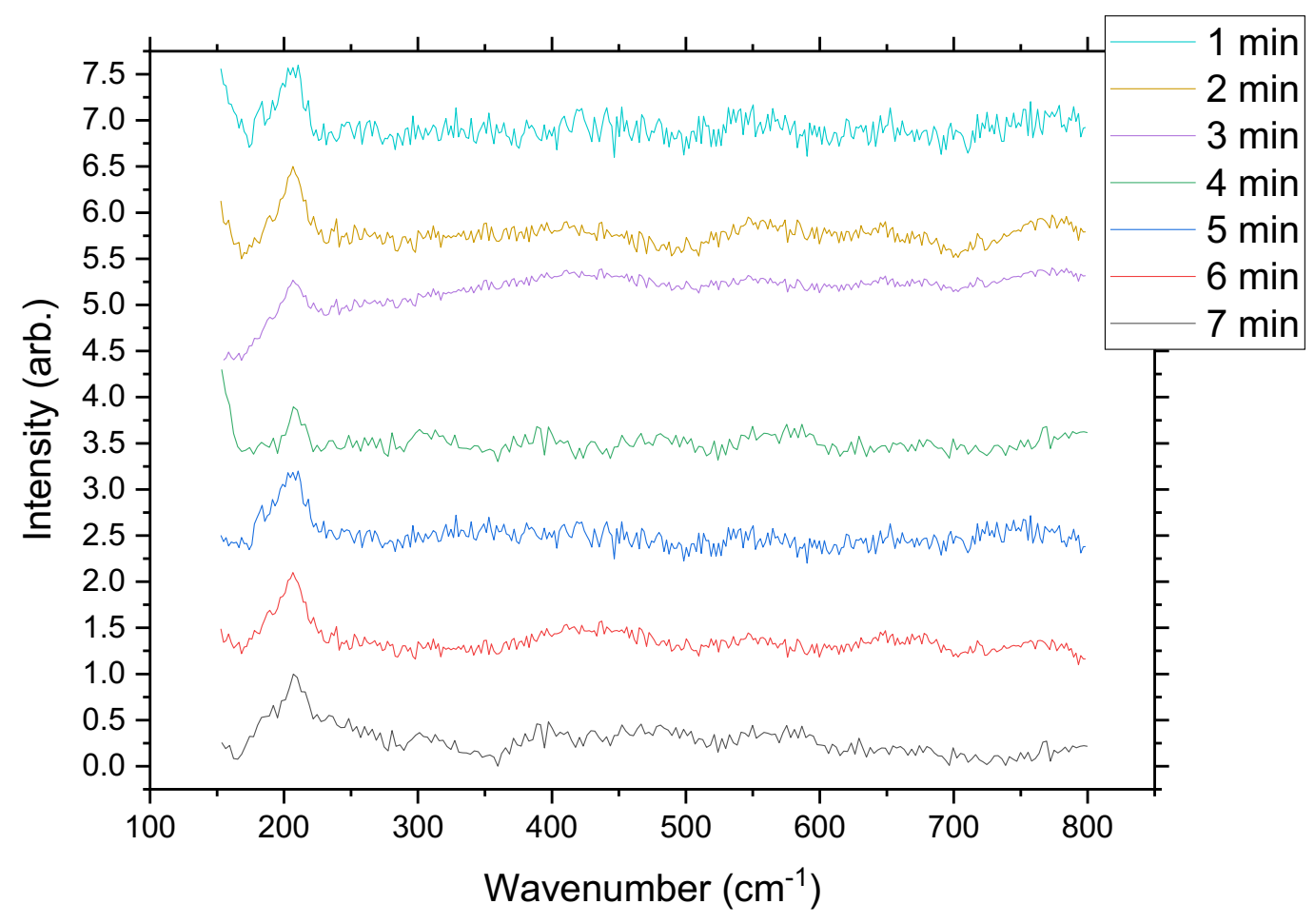

Figure S9: Resonant Raman spectra of partially etched nanoplatelets $1-7$ min after addition of the etechant. In all cases coupling with a LO-phonon mode at $200 \mathrm{~cm}^{-1}$ is observed. 
S10: Temperature dependent absorption and emission of nanoplatelets and nanorings

Figure S10 shows the photoluminescence excitation spectra of nanorings and nanoplatelets. The spectra mirror the absorption line shape, suggesting all excitations end up in the excitonic or defect states.

a

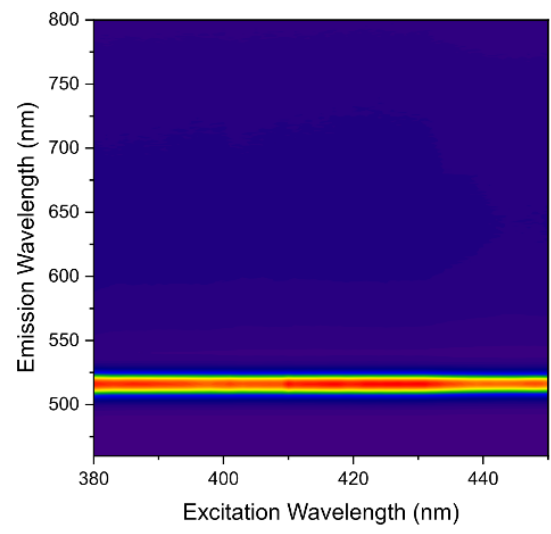

b

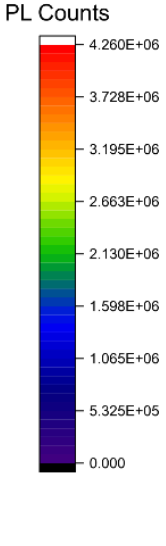

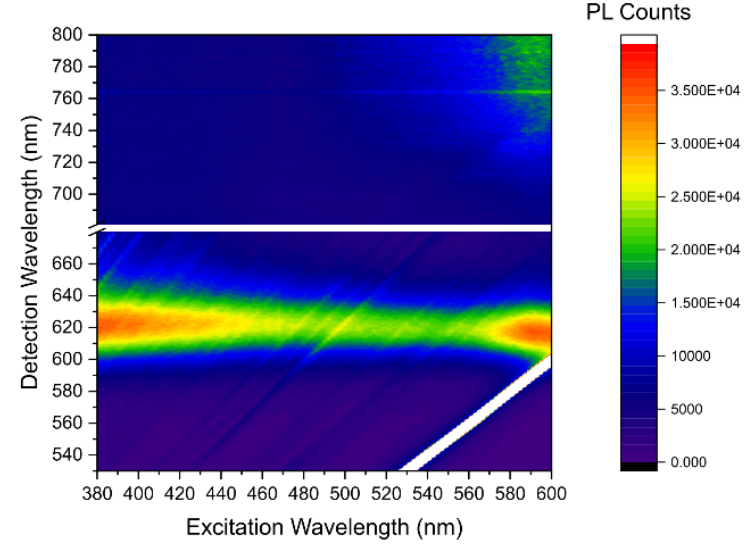

Figure S10: a-b. Photoluminescence excitation spectrum of nanoplatelets (left) and nanorings (right).

Figure S10i shows the ratio of integrated photoluminescence from the excitonic versus defect state as a function of temperature for nanorings and nanoplatelets. In the case of nanoplatelets there remains $\sim 10$ times greater emission from the excitonic state as a compared to trap states, whereas in nanorings there is almost equal amounts of trap versus defect emission on reaching $4 \mathrm{~K}$.

a

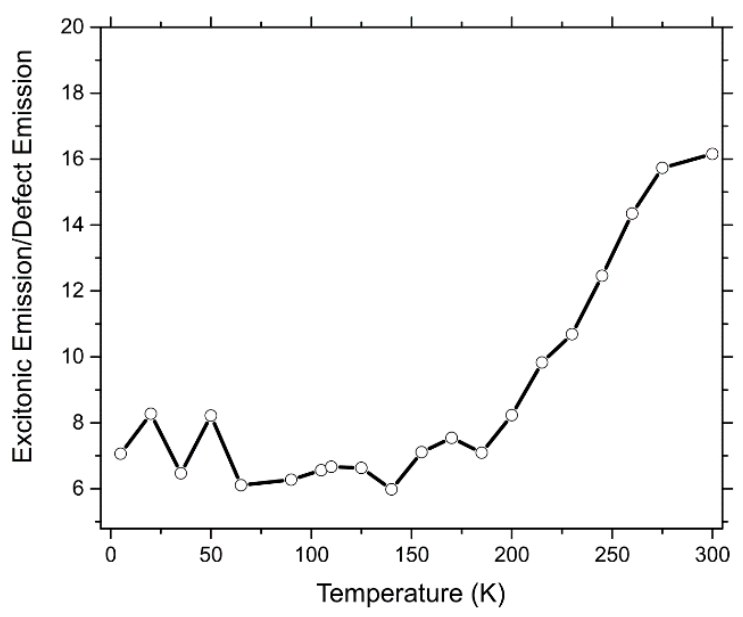

b

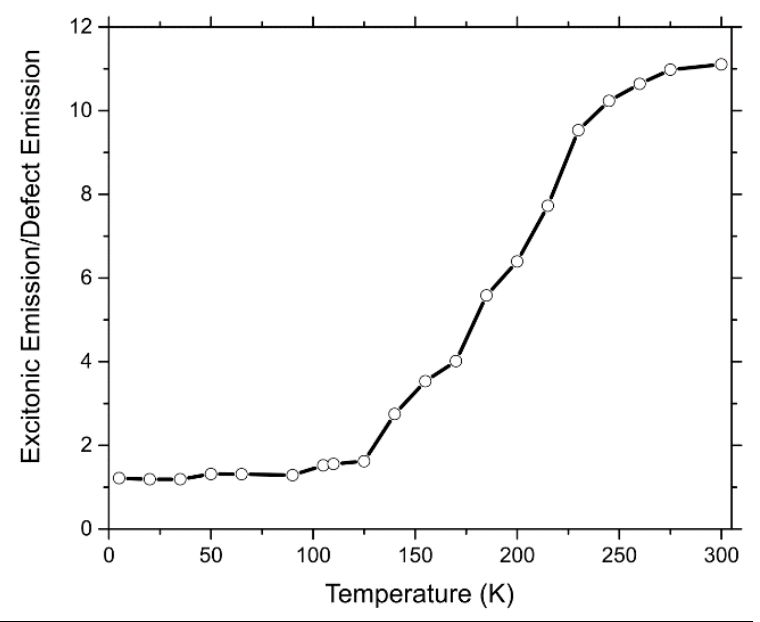

Figure S10i a-b. Ratio of integrated photoluminescence from excitonic state versus defect state as a function of temperature for nanorings (left) and nanoplatelets (right). 
Figure S10ii shows the cryogenic absorption spectrum of nanoplatelets and nanorings plotted on a logarithmic scale to emphasise the initial blue shift to higher energies of the excitonic absorption peak followed by a shift to lower energies on cooling below $\sim 100-150 \mathrm{~K}$.

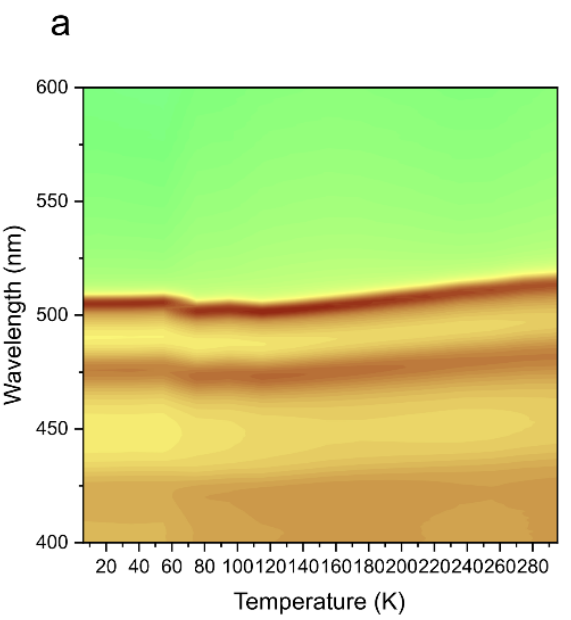

b
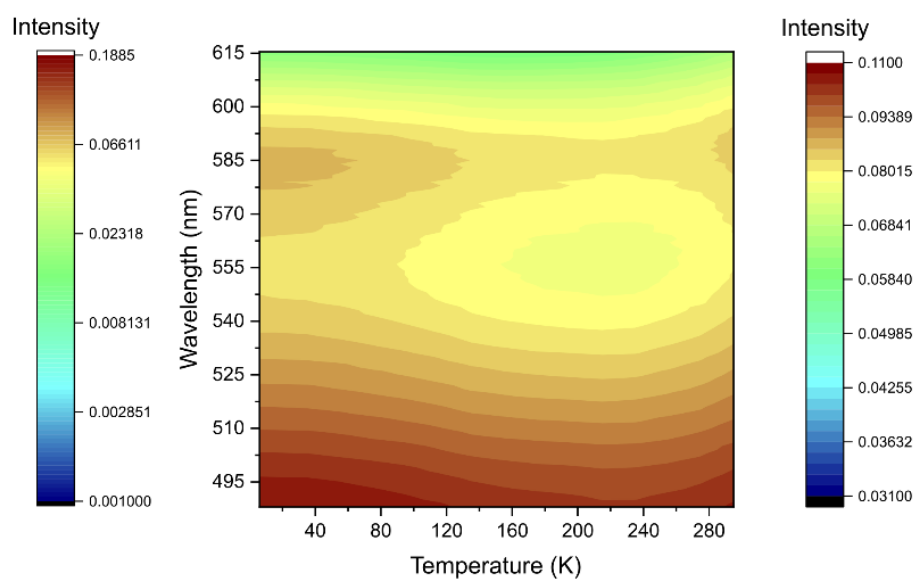

Figure S10ii: a-b. Cryogenic absorption spectrum of nanoplatelets (left) and nanorings (right). Both are plotted on a logarithmic scale.

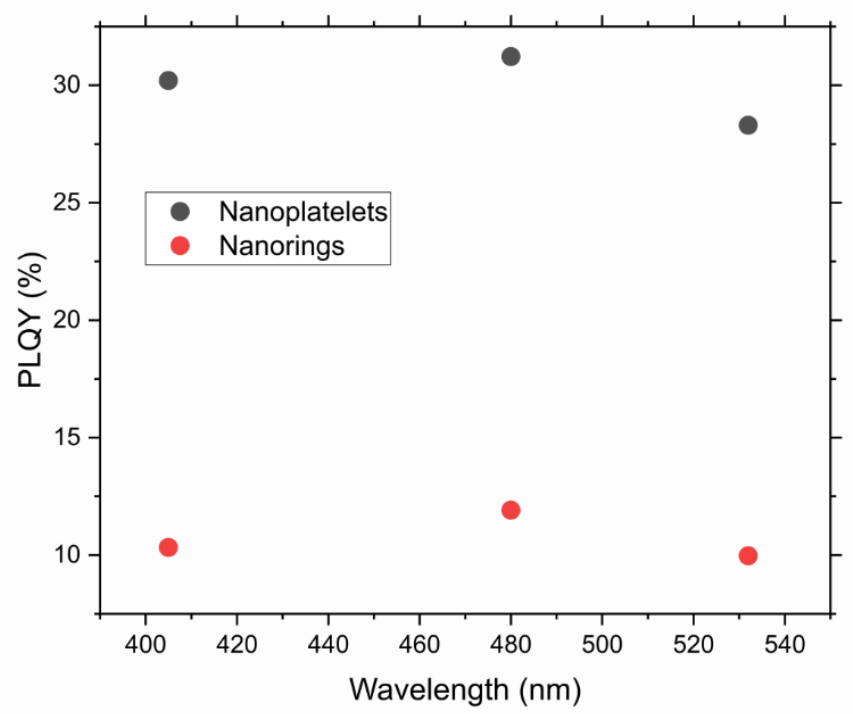

Figure S10iii: PLQY of nanoplatelets and nanorings as a function of laser excitation wavelength. There is no dependence of the PLQY on excitation energy suggesting hot carrier trapping effects are minimal ${ }^{12}$. 
S11: Cryogenic transient photoluminescence spectra of nanoplatelets and nanorings

Measuring the time resolved emission spectra of nanorings as a function of temperature gives additional insight into the band edge exciton structure. Figure S11 and S11i show the transient PL response as a function of temperature (PL integrated along man exciton peak). A biexponential fit is made to the early time decay (0-1.5 ns only recorded due to limitations of streak camera setup). This shows a complex non-monotonic variation with temperature (see main text for further discussion).

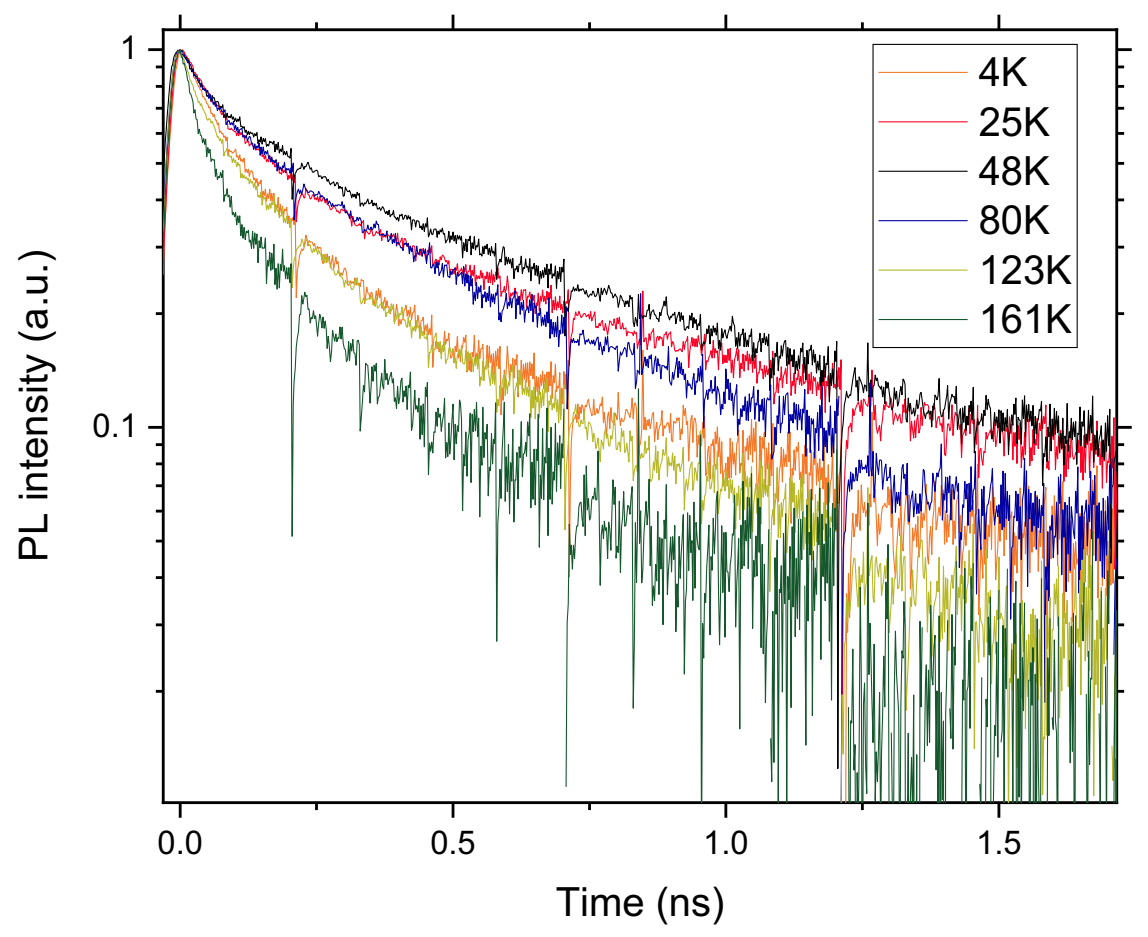

Figure S11: Transient PL spectra of CdSe nanorings as a function of temperature. The decay shows a non-monotonic variation with temperature.
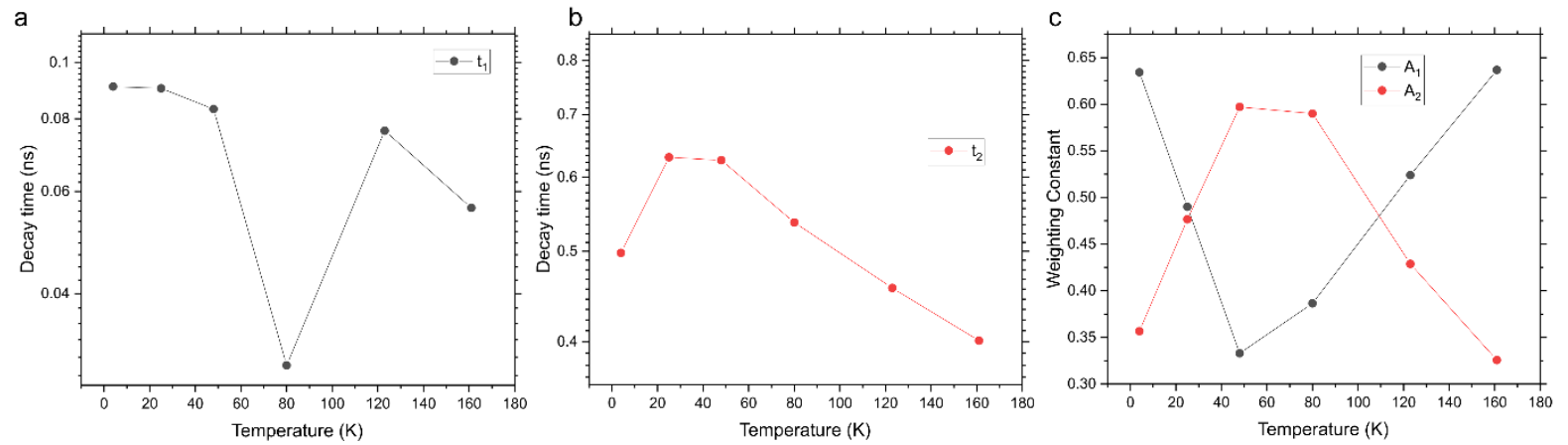

Figure S11i: a. The transient PL response in the 0-1.5 ns range is fit with a biexponential decay. The fast component $t_{1}$ initially decreases with temperature in the $186 \mathrm{~K}$ to $80 \mathrm{~K}$ range, before increasing slightly on lowering to $4 \mathrm{~K}$. The decay time lies between $0.03-0.1 \mathrm{~ns}$ in the $4-160 \mathrm{~K}$ temperature range. b. The $\mathrm{t}_{2}$ decay component rises with temperature from $0.4 \mathrm{~ns}$ at $160 \mathrm{~K}$ to $0.65 \mathrm{~ns}$ at $60 \mathrm{~K}$. The decay 
time then decreases to $0.5 \mathrm{~ns}$ on further cooling to $4 \mathrm{~K}$. c. Weighting coefficients for $t_{1}$ and $t_{2}$. The weight of $t_{1}$ initially drops with temperature before rising at after $60 \mathrm{~K} ; \mathrm{t}_{2}$ on the hand follows the opposite trend. 
S12: Modelling surface emission from nanoplatelets and naorings

To analyse the temperature dependence of the relative intensities of the narrow 'core' and broad 'surface' PL, a semi-classical Marcus-Levich-Jortner electron transfer model was constructed as per previous studies. ${ }^{13,14}$

The forward electron transfer rate, from 'core' to 'surface', was defined as $W$ :

$$
W=A e^{-S(2 \bar{n}+1)} I_{p}\left(2 S\left(\bar{n}(\bar{n}+1)^{\frac{1}{2}}\right)\right)\left[\frac{\bar{n}+1}{\bar{n}}\right]^{\frac{p}{2}} \text { (Equation E4) }
$$

where the constant $A=10^{13}, I_{p}(x)$ is the modified Bessel function of the first kind (imaginary) of order $p . \mathrm{S}$ is the Huang Rhys parameter, defined as:

$$
S=\frac{\lambda}{h \omega_{e f f}}(\text { Equation E5) }
$$

The value of $\omega$ is $200 \mathrm{~cm}^{-1}$ and represents the CdSe longitudinal optical (LO) phonon mode and can be converted to $\omega_{\text {eff }}$ by multiplication by $c$. The constants $h$ and $k$ are Planck's constant and the Boltzmann constant respectively. Here $\lambda$ is the internal reorganization energy for CdSe nanocrystals previously found to be approximately $0.6 \mathrm{eV},{ }^{15}$ which we apply to the nanoplatelets and nanorings.

$\bar{n}$ is defined as the average phonon occupation number:

$$
\bar{n}=\left(e^{\left(\frac{h \omega}{k T}\right)}-1\right)^{-1}(\text { Equation E6) }
$$

$p$ is defined as:

$$
p=\frac{\Delta G}{h \omega_{e f f}}(\text { Equation E7) }
$$

The Boltzmann factor $B$ was defined as:

$$
B=e^{\frac{\Delta G}{k T}}(\text { Equation E8) }
$$

The steady-state temperature-dependent PL spectra for nanorings and nanoplatelets were first converted into lineshapes for population analysis. This was done by performing the $x$-axis Jacobian correction from $\mathrm{nm}$ to $\mathrm{eV}$ followed by multiplication by $v^{3} .{ }^{16}$ The integrated lineshapes for the high energy 'core' and lower energy 'surface' peaks were then compared to the relative populations of their respective states. A series of coupled differential equations to describe the 3-state kinetic model was used, which were all set to zero to represent the steady-state condition, along with a normalisation factor:

$$
\frac{d n_{0}}{d t}=-\frac{n_{0}}{\tau_{0}}+\frac{n_{1}}{\tau_{1}}+\frac{n_{2}}{\tau_{2}}=0
$$




$$
\begin{aligned}
& \frac{d n_{1}}{d t}=\frac{n_{0}}{\tau_{0}}-\frac{n_{1}}{\tau_{1}}-W n_{1}+W B n_{2}=0 \\
& \frac{d n_{2}}{d t}=W n_{1}-W B n_{2} \frac{n_{2}}{\tau_{2}}=0 \\
& n_{0}+n_{1}+n_{2}=1 \quad \text { (Equation E9) }
\end{aligned}
$$

The room-temperature PL lifetimes for the 'core' and 'surface' states were measured by spectrally resolved TCSPC fitted to triexponentials and used in the model as $\tau_{1}$ and $\tau_{2}$ respectively. The equations were simultaneously solved for the populations using the $f$ solve function in MATLAB R2016b. All units were converted to ns to minimise numerical errors. The populations were related to emission intensities through division by respective emission lifetime. A one dimensional optimisation of $\Delta \mathrm{G}$ was performed by a grid search using the mean absolute error to the measured integrated lineshapes.

For both nanoplatelets and nanorings, the value of $\sim 0.024 \mathrm{eV}$ for $\Delta \mathrm{G}$ obtained the best fit, shown below. The model accurately represented the temperature dependence of the relative 'core' and 'surface' emissions at higher temperatures for both nanoplatelets and nanorings. However, at lower temperatures, the observed intensities deviated from the model. One effect not accounted for in the model was the nonlinear temperature-dependence of the observed radiative lifetimes observed through cryo-TCSPC. As the steady state relative populations are highly dependent on the lifetimes, it is likely the model cannot fully account for the observed populations at lower temperatures. The presence of additional phonon modes other than the $200 \mathrm{~cm}^{-1}$ LO-phonon at low temperatures are not accounted for, which may also contribute to deviation from the model. Indeed, as shown via our cryogenic TCSPC (SI, S11) measurements the nanoring PL shows a complex non-monotonic behaviour with temperature. This suggests thermal mixing between several band-edge exciton states, preventing accurate fitting of the PL data with the above model. Expanding the electron transfer model to include these multiple states is beyond the scope of this work but should be the focus of future studies. 

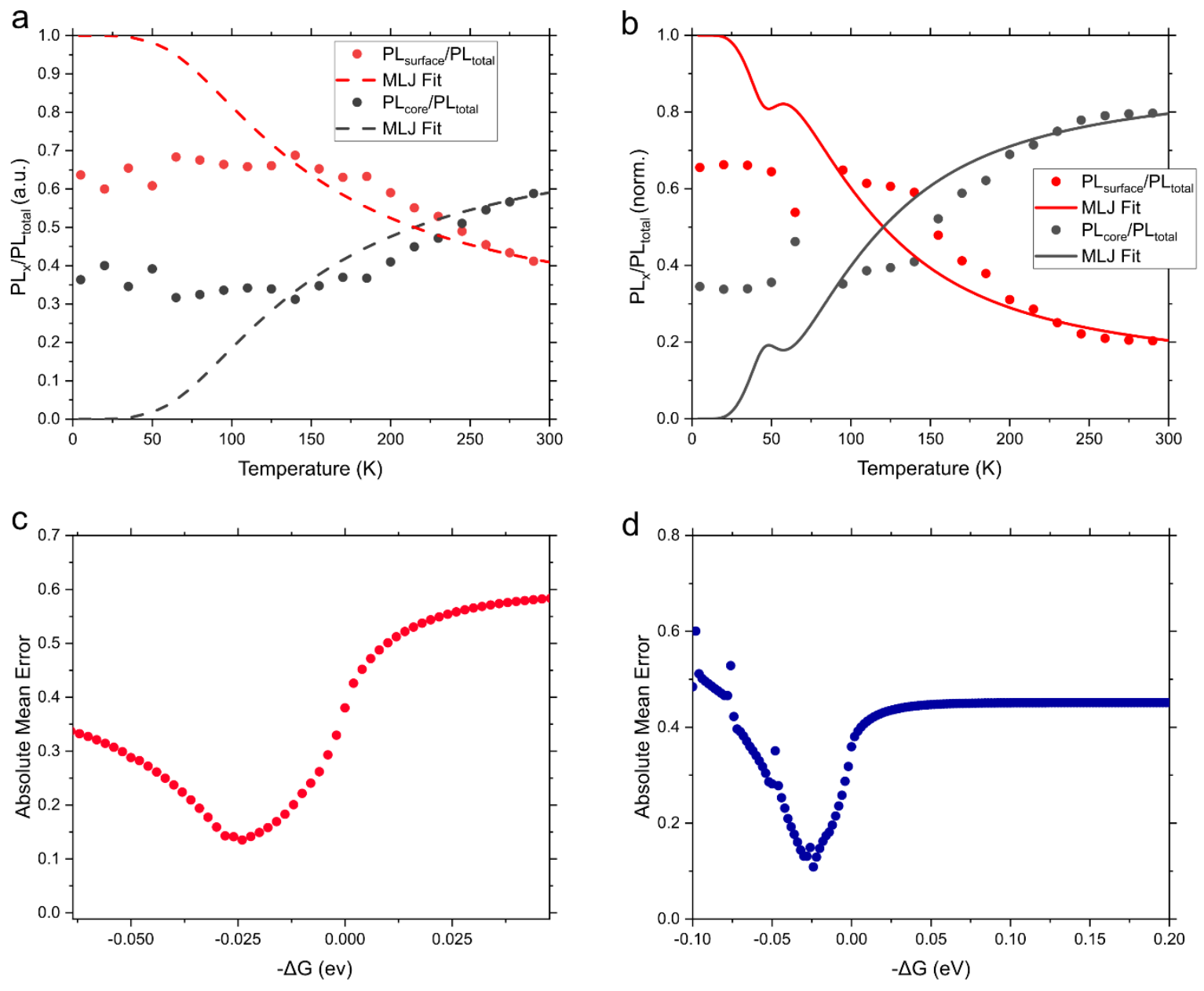

Figure S12: a. Fit of $\mathrm{PL}_{x} / \mathrm{PL}_{\text {total }}$ of nanoplatelets where $x$ is surface or core PL to Marcus-Levich-Jortner model represented by equation E9. At high temperatures the model fits the data well but deviates at lower temperatures. b. Fit of $\mathrm{PL}_{x} / \mathrm{PL}_{\text {total }}$ of nanorings where $x$ is surface or core PL to Marcus-LevichJortner model represented by equation E9. At high temperatures the model fits the data well but deviates at lower temperatures. c. Absolute mean error as function of $\Delta \mathrm{G}$ parameter for nanoplatelets, obtained following grid search. d. Absolute mean error as function of $\Delta \mathrm{G}$ parameter for nanorings obtained following grid search. 
S13: Room temperature transient photoluminescence spectra of nanoplatelets and nanorings

Measuring the transient photoluminescence spectra from nanoplatelets and nanorings reveals an additional emissive state centred around $530-550 \mathrm{~nm}$ and $740 \mathrm{~nm}$ respectively. This state which also appears in the steady state photoluminescence spectra grows in intensity at low temperatures. In nanoplatelets the emission from this state is relatively long-lived $\tau_{\mathrm{av}} \sim 140 \mathrm{~ns}$ as compared to nanorings where the decay rate is similar to that assigned to emission from the excitonic state.

Tables T2 and T3 summarise the decay constants and weighting coefficients obtained from multiexponential fitting of the wavelength resolved TCSPC data for nanoplatelets and nanorings. The decay is fit using equation $\mathrm{E} 1$.

\begin{tabular}{|l|l|l|l|}
\hline $505 \mathrm{~nm}$ & $510 \mathrm{~nm}$ & $515 \mathrm{~nm}$ & $540 \mathrm{~nm}$ \\
\hline $0.34 \pm 0.1 \mathrm{~ns}(0.93)$ & $0.39 \pm 0.1 \mathrm{~ns}(0.92)$ & $0.36 \pm 0.2 \mathrm{~ns}(0.91)$ & $1.5 \pm 0.6 \mathrm{~ns}(0.31)$ \\
\hline $28.6 \pm 2 \mathrm{~ns}(0.11)$ & $28.7 \pm 2 \mathrm{~ns}(0.12)$ & $27.3 \pm 2 \mathrm{~ns}(0.13)$ & $50.3 \pm 3 \mathrm{~ns}(0.22)$ \\
\hline $94.3 \pm 2 \mathrm{~ns}(0.11)$ & $95.1 \pm 2 \mathrm{~ns}(0.13)$ & $96.1 \pm 2 \mathrm{~ns}(0.14)$ & $162.8 \pm 3 \mathrm{~ns}(0.44)$ \\
\hline
\end{tabular}

Table T2: Summary of individual rate constants and associated uncertainty for wavelength resolved TCSPC data of nanoplatelets. The brackets indicate the respective weighting coefficients of the decay.

\begin{tabular}{|l|l|l|l|}
\hline $570 \mathrm{~nm}$ & $600 \mathrm{~nm}$ & $630 \mathrm{~nm}$ & $740 \mathrm{~nm}$ \\
\hline $0.7 \pm 0.3 \mathrm{~ns}(0.63)$ & $1.3 \pm 0.2 \mathrm{~ns}(0.52)$ & $1.8 \pm 0.2 \mathrm{~ns}(0.59)$ & $2.5 \pm 0.6 \mathrm{~ns}(0.81)$ \\
\hline $5.8 \pm 2 \mathrm{~ns}(0.27)$ & $7.2 \pm 2 \mathrm{~ns}(0.34)$ & $11.7 \pm 2 \mathrm{~ns}(0.31)$ & $9.3 \pm 5 \mathrm{~ns}(0.1)$ \\
\hline $30.1 \pm 1 \mathrm{~ns}(0.17)$ & $40.3 \pm 2 \mathrm{~ns}(0.11)$ & $54.8 \pm 2 \mathrm{~ns}(0.24)$ & $62.8 \pm 1 \mathrm{~ns}(0.21)$ \\
\hline
\end{tabular}

Table T3: Summary of individual rate constants and associated uncertainty for wavelength resolved TCSPC data of nanorings. The brackets indicate the respective weighting coefficients of the decay.

Stretched exponential functions and variations thereof can be used to obtain insight into carrier trapping in nanocrystals. ${ }^{17-19}$ Here we survey a few commonly used equations and evaluate their response for nanoplatelets and nanorings. We additionally outline limitations of the models and emphasise their use to be of qualitative significance in agreeing with many of our experimental observations.

The transient PL response of nanorings and nanoplatelets can be fit to obtain an estimate of the mean number of traps per nanoparticle $(\bar{N})$ and trapping $\left(k_{l}\right)$ and detrapping $\left(k_{2}\right)$ rates by charges. This is done using the following equation ${ }^{18}$ : 


$$
I(t)=\sum_{N} e^{-N} \frac{\bar{N}^{N}}{N !}\left(A e^{-\left(\alpha_{1} \frac{t}{\tau}\right)}+B e^{-\left(\alpha_{2} \frac{t}{\tau}\right)}\right) \text { (Equation E10) }
$$

Here, $N$ is the number of identical electron (or hole) traps, $\tau$ is the decay time constant, $\alpha_{1,2}$ and, $A$ and $B(B=1-A)$, are time and weighting constants related to the trapping and de-trapping rates. Explicitly:

$$
\begin{gathered}
\alpha_{1,2}=\frac{1}{2}\left(1+N K_{1}+K_{2}+ \pm \sqrt{\frac{1}{4}\left(1+N K_{1}+K_{2}\right)^{2}-K_{2}} \quad\right. \text { (Equation E11) } \\
A=\frac{1+N K_{1}-\frac{1}{2}\left(1+N K_{1}+K_{2}+ \pm \sqrt{\frac{1}{4}\left(1+N K_{1}+K_{2}\right)^{2}-K_{2}}\right.}{2 \sqrt{\frac{1}{4}\left(1+N K_{1}+K_{2}\right)^{2}-K_{2}}} \text { (Equation E12) }
\end{gathered}
$$

We note that $K_{1}=k_{1} \tau$ and $K_{2}=k_{2} \tau$

In addition, a trap activation energy can be estimated from:

$$
\frac{K 2}{K 1}=e^{\left(-\frac{\Delta E}{k T}\right)}(\text { Equation E13) }
$$

Fitting equation E10 to the transient PL data produces the values shown in Table T4.

\begin{tabular}{|l|l|l|l|l|}
\hline & \multicolumn{1}{|c|}{$\bar{N}$} & \multicolumn{1}{|c|}{$k_{l}\left(\mathrm{~s}^{-1}\right)$} & \multicolumn{1}{c|}{$k_{2}\left(\mathrm{~s}^{-1}\right)$} & $\Delta \mathrm{E}(\mathrm{meV})$ \\
\hline Nanorings & 14.9 & $9.3 \times 10^{8}$ & $1.4 \times 10^{7}$ & 11 \\
\hline Nanoplatelets & 5.2 & $1.3 \times 10^{7}$ & $1.7 \times 10^{6}$ & 28 \\
\hline
\end{tabular}

Table T4: Summary of fitting constants obtained using equation E10 as model for trapping and detrapping on nanoring/nanoplatelet surfaces.

The above model treats the nanoparticles as a two-level system with a ground state and an excited emissive (bright) state. As cryogenic transient PL measurements show this hypothesis is unlikely to be valid at least for the case of the nanorings. The model is also based around interactions between the nanocrystals and assumes that there may be some inhomogeneity in the passivation of nanoparticles. Although this latter point may be true, measurements here were all performed in solution where we assume there is little stacking interactions between nanocrystals. Consequently, the outputs of the model 
should be treated with caution as charge tunnelling and other inter-particle effects are likely to be weak here. They do qualitatively show that the average number of traps on nanorings to be three times larger than for nanoplatelets in keeping with our observations.
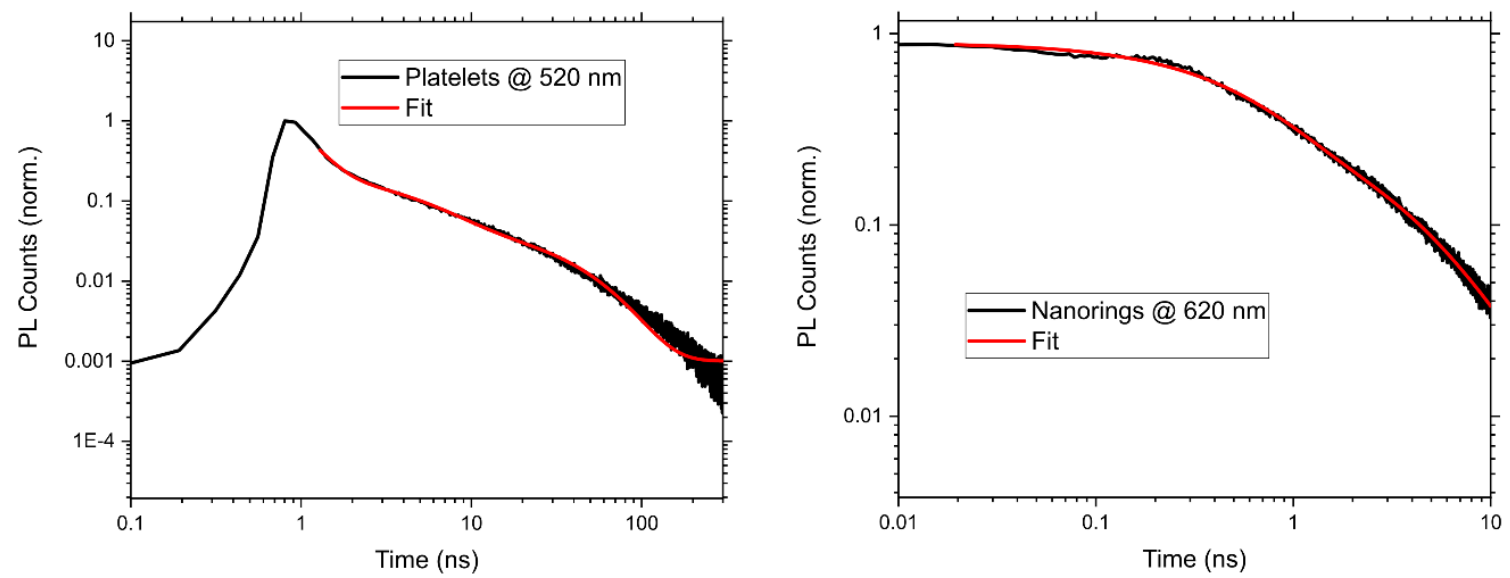

Figure S13: Fitting of equation E10 to PL decay on nanoplatelets (left) and nanorings (right). Black line shows raw data and red shows fit.

A second, simpler stretched exponential expression can also be used to fit the PL decays namely: ${ }^{17-19}$

$$
I(t)=e^{-\frac{t}{\tau}} e^{-a\left(\frac{t}{\tau}\right)^{\beta}}(\text { Equation E14) }
$$

$\beta$, the stretching parameter, is the key term in this equation and has physically been used to assess the nature of energy transfer between quantum dots or excitons and trapping sites in nanocrystals. When $\beta$ $<0.5$, it is often taken as a sign of PL quenching by lower energy acceptor nanoparticles via FRET, conversely for $\beta>0.5$ it has been shown quenching by surface charges is often important. Fitting equation E14 gives $\beta$ values of 0.63 and 0.82 for nanoplatelets and nanorings respectively. This is in keeping with some PL quenching by charge states on the surface of nanoplatelets and nanorings and weak interparticle PL transfer. ${ }^{17}$ 

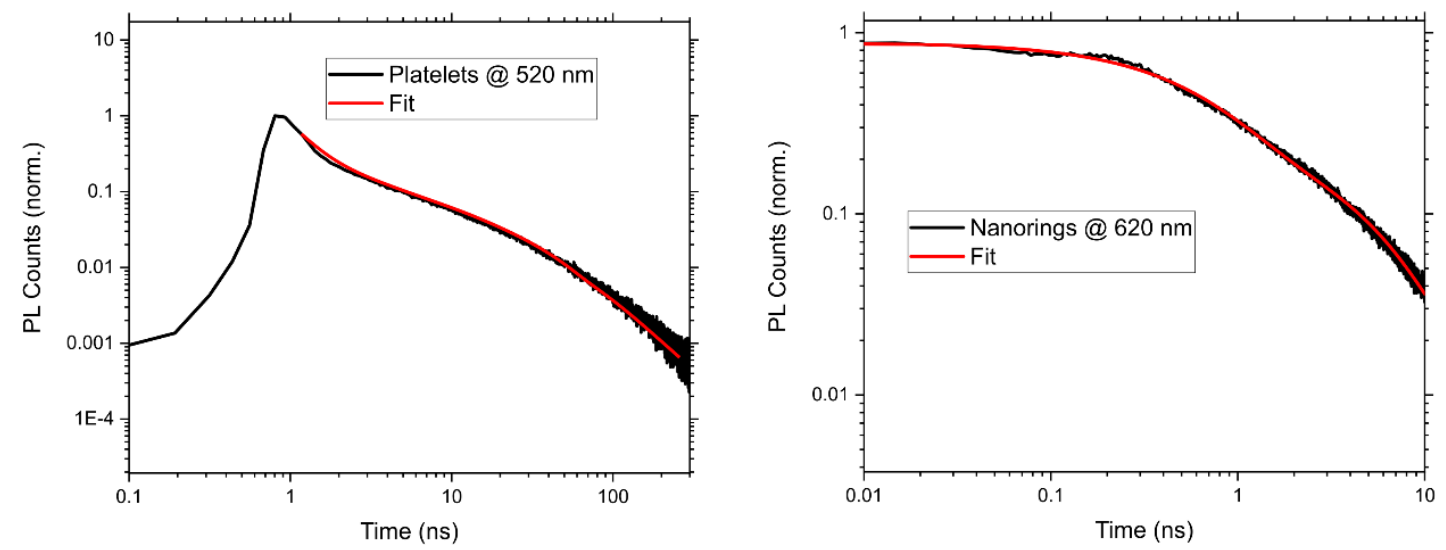

Figure S13i: Fitting of equation E14 to PL decay on nanoplatelets (left) and nanorings (right). Black line shows raw data and red shows fit.

Measuring the time resolved and steady state PL of nanorings at two different concentrations in hexane shows no difference in the PL lifetimes or spectral features (Figure S13ii and S13iii). This suggests concentration based PL quenching e.g. via stacking is not significant in these materials.

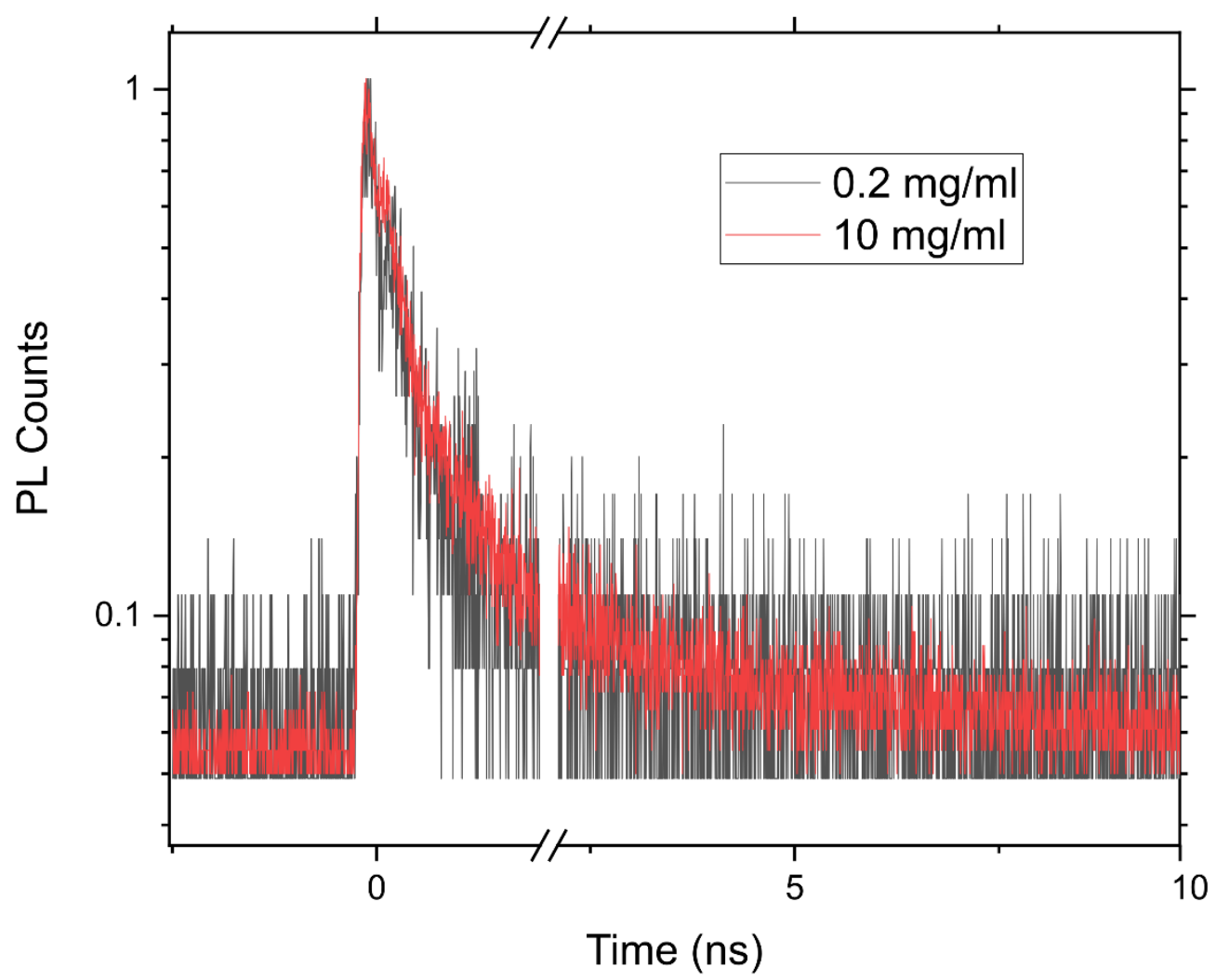

Figure S13ii: PL decay (at excitonic peak $\sim 60 \mathrm{~nm}$ ) of nanorings of $10 \mathrm{mg} / \mathrm{mL}$ concentration (red) and $0.2 \mathrm{mg} / \mathrm{mL}$ concentration in hexane. Diluting the nanorings concentration by a factor of 5 does not alter the PL decay rate, suggesting that concentration based stacking and PL quenching of nanorings is not significant. 


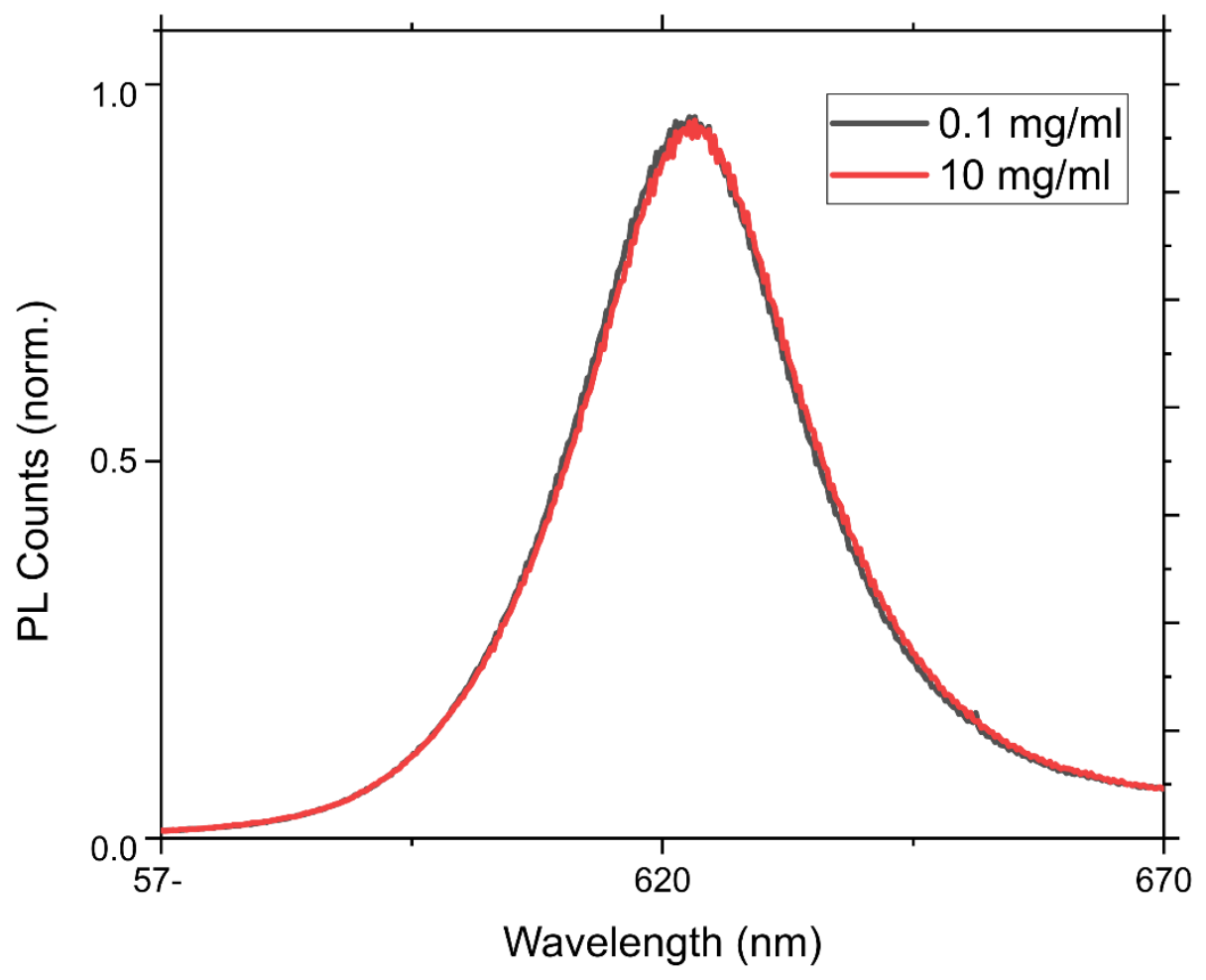

Figure S13iii: Steady state PL spectrum of nanorings of $10 \mathrm{mg} / \mathrm{mL}$ concentration (red) and $0.1 \mathrm{mg} / \mathrm{mL}$ concentration in hexane. Diluting the nanorings concentration by a factor of 10 does not alter the PL spectrum shape or introduce new peaks. This suggests that concentration based stacking is not significant. 
S14: Kelvin probe contact potential difference

The Kelvin probe is sensitive to out of plane charge changes, particularly the net surface dipole via measurement of the contact potential difference (CPD; - the difference in work function between sample and tip), hence the orientation, etc. of nanostructures on the substrate is also an important consideration. Only nanoparticles $\sim 2 \mathrm{~nm}$ from the top surface, i.e. approximately the thickness of a single 4-monolayer nanoplatelet or nanoring will contribute to the KP signal. Depending on the orientation of nanoplatelets or nanorings gases may diffuse into and out of the particles at different rates owing to the difference in surface area coverage for vertical versus horizontally stacked platelets, as well as the chemistry of different facets. From TEM measurements (SI, S1) we expect most particles to lie flat, albeit with some overlap; samples were prepared from sufficiently low concentration solutions such as to expect monolayer coverage. As a result, we expect any changes in CPD reported to be an upper bound.

In the case of both nanoplatelets and nanorings we see the CPD is dramatically altered by the introduction of oxygen to the ambient environment (Figure S14a). For nanoplatelets the oxygen alters the contact potential difference (whose change is proportional to the change in net surface dipole) by $\sim 300 \mathrm{mV}$ and in nanorings by $\sim 600 \mathrm{mV}$. Interestingly in both nanostructures, after the sudden increase in oxygen concentration, the rate of change in CPD has a biphasic response with an initial fast change and then a slower secondary increase. The initial change in CPD is likely due to incorporation of oxygen via electronic bonding. Conversely the second regime likely corresponds to diffusion of surface oxygen, potentially through weak van der Waals interactions. This is supported by the recovery of CPD through the introduction of nitrogen into the system. In nanoplatelets, this second phase does not involve a pronounced change in the $\mathrm{CPD}$, with the $\mathrm{CPD}$ returning to almost the same value on refilling the chamber with nitrogen; this suggests that oxygen incorporation is much weaker. These results are consistent with studies of biphasic, reversible, PL enhancement by oxygen in nanoplatelets. There, it was shown oxygen passivates excess electrons at the nanoplatelet surface and reduces the sensitivity of holes to surface defects. This in-turn promotes radiative recombination leading to brighter emission. The biphasic response was, as here, ascribed to static and dynamic quenching regimes, where, in the latter case, gas adsorption deactivates less efficient surface traps. The larger overall CPD change in nanorings is consistent with surface defects not being as well passivated in these materials by e.g. organic ligands, thus allowing interstitial $\mathrm{O}_{2}$ to have a more pronounced effect. The fact that the CPD does not return to the same value on removal of oxygen suggests the $\mathrm{O}_{2}$ has caused partially irreversible physio-chemical change to the nanoring surface.

In addition to the effect of oxygen we also investigated the effect of light exposure on the CPD by measuring the CPD following illumination and extinction. In both materials the CPD changes quite 
dramatically $(\sim 40 \mathrm{mV})$ on light soaking. In the case of nanoplatelets, the recovery of the CPD is relatively slow as compared to the nanorings. This slow return of the CPD is ascribed to the reduced rate of freeing of charges from trap sites in the nanostructure and suggests that nanoplatelets contain either, a greater number of, or deeper traps. ${ }^{21,23,24}$ This is in agreement with the low temperature PL measurements which show that the trap degeneracy (from Equation 1) in nanoplatelets is higher than in nanorings, and also confirmed by the slightly lower Urbach energy for nanorings over nanoplatelets.
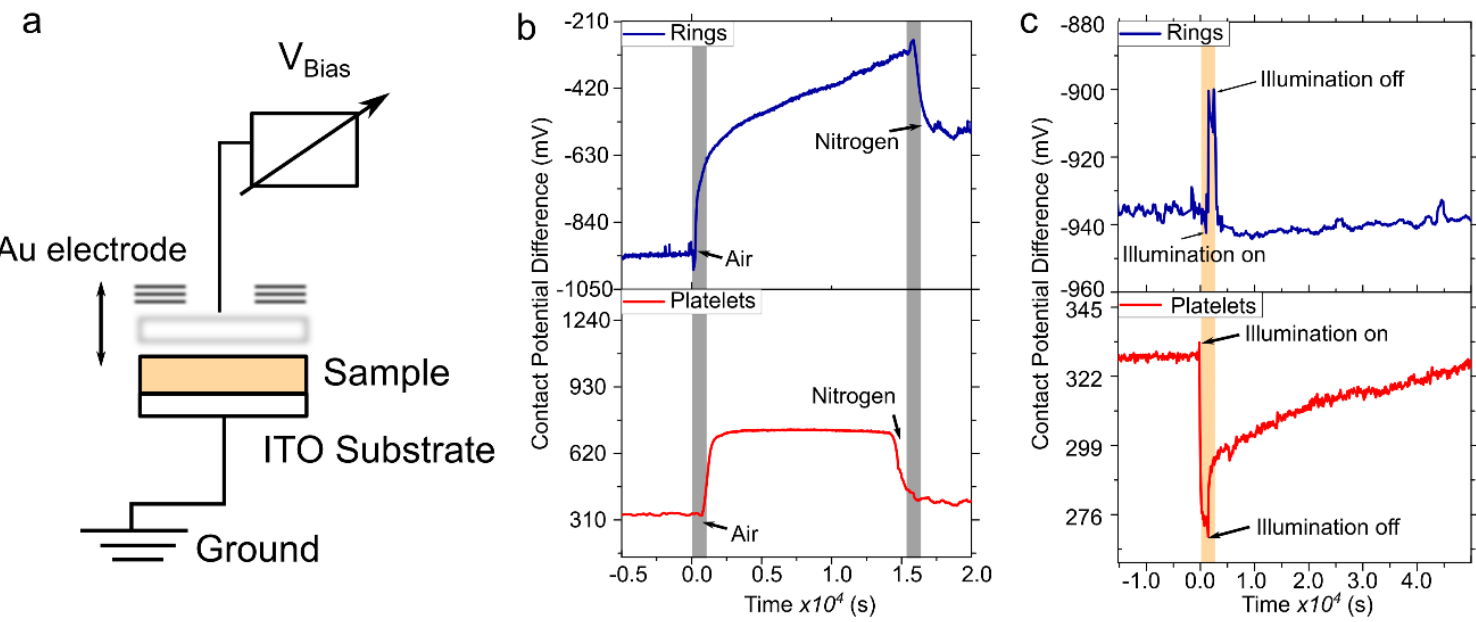

Figure S14: Kelvin probe study of oxygen dependence and surface photovoltage response of nanoplatelets and nanorings. a. Schematic of Kelvin probe setup. In the Kelvin probe contact potential difference (CPD) measurement, a vibrating gold tip (potential $V_{\mathrm{b}}$ ) is suspended a distance above a sample on a grounded ITO substrate. The peak-to peak amplitude of the time-varying voltage from the tip is proportional to the sum of $\mathrm{V}_{\mathrm{b}}$ and the contact potential difference $\mathrm{V}_{\mathrm{cpd}}$ between the inner face of the vibrating tip and the sample surface. As $\mathrm{V}_{\mathrm{b}}$ is altered to minimise the measured voltage, $\mathrm{V}_{\mathrm{cpd}}$ can be determined ${ }^{20} \mathbf{b}$. The initial dark contact potential difference (CPD) between the stainless steel tip in nitrogen atmosphere is $-940 \mathrm{mV}$ and $+340 \mathrm{mV}$ for the nanorings and nanoplatelets respectively. In the case of the nanorings and platelets, a rapid increase in work function on the order of $300 \mathrm{mV}$ is observed upon exposure to ambient atmosphere as it is pumped into the system at $\mathrm{t}=0$. This abrupt change is attributed to the formation of a surface dipole as oxygen is absorbed into the active surface of nanomaterials. ${ }^{21}$ In the nanorings, the transition into a linear CPD change, between $-600 \mathrm{mV}$ to $300 \mathrm{mV}$, likely represents a further diffusion of oxygen into the rings. At 30 minutes, the atmosphere is replaced with nitrogen and the CPD recovers to $-600 \mathrm{mV}$. This indicates oxygen can be partially removed from physisorbed sites, however the initial binding to oxygen is beyond thermally activated desorption at room temperature. c. Surface photovoltage response comparison between rings and platelets in nitrogen. Thin films of samples were held in a dark nitrogen environment then exposed to an AM1.5 white light source for $30 \mathrm{~s}$ while the recovery of the CPD is monitored. The magnitude of the SPV response is on the order of $40 \mathrm{mV}$ for both rings and platelets. As, in general, the SPV recovery is trap-dominated we can infer the number and energy depth of trap states depending the CPD change after exposure to white light. ${ }^{21,22}$ We find for the rings that the SPV response decays within the instrument time resolution $(<1 \mathrm{~s})$, whereas for the platelets this is a much longer process $(>300 \mathrm{~s})$. This suggests that the traps are deeper and longer lived in the rings compared to platelets. 
References

(1) Page, R. C.; Espinobarro-Velazquez, D.; Leontiadou, M. A.; Smith, C.; Lewis, E. A.; Haigh, S. J.; Li, C.; Radtke, H.; Pengpad, A.; Bondino, F.; et al. Near-Unity Quantum Yields from Chloride Treated CdTe Colloidal Quantum Dots. Small 2015, 11 (13), 1548-1554. https://doi.org/10.1002/smll.201402264.

(2) Mondal, N.; De, A.; Samanta, A. Achieving Near-Unity Photoluminescence Efficiency for Blue-Violet-Emitting Perovskite Nanocrystals. ACS Energy Lett. 2019, 4 (1), 32-39. https://doi.org/10.1021/acsenergylett.8b01909.

(3) Pandya, R.; Chen, R. Y. S.; Cheminal, A.; Dufour, M.; Richter, J. M.; Thomas, T. H.; Ahmed, S.; Sadhanala, A.; Booker, E. P.; Divitini, G.; et al. Exciton-Phonon Interactions Govern Charge-Transfer-State Dynamics in CdSe/CdTe Two-Dimensional Colloidal Heterostructures. J. Am. Chem. Soc. 2018, 140 (43), 14097-14111. https://doi.org/10.1021/jacs.8b05842.

(4) Hamizi, N. A.; Johan, M. R. Optical Properties of CdSe Quantum Dots via Non-TOP Based Route. Int. J. Electrochem. Sci. 2012, 7, 8458-8467.

(5) Jana, S.; Phan, T. N. T.; Bouet, C.; Tessier, M. D.; Davidson, P.; Dubertret, B.; Abécassis, B. Stacking and Colloidal Stability of CdSe Nanoplatelets. Langmuir 2015, 31 (38), 1053210539. https://doi.org/10.1021/acs.langmuir.5b02152.

(6) DeLong, K. W.; Trebino, R.; Hunter, J.; White, W. E. Frequency-Resolved Optical Gating with the Use of Second-Harmonic Generation. J. Opt. Soc. Am. B 1994, 11 (11), 2206-2215.

(7) García-Santamaría, F.; Chen, Y.; Vela, J.; Schaller, R. D.; Hollingsworth, J. A.; Klimov, V. I. Suppressed Auger Recombination in "Giant" Nanocrystals Boosts Optical Gain Performance. Nano Lett. 2009, 9 (10), 3482-3488. https://doi.org/10.1021/n1901681d.

(8) Hou, X.; Kang, J.; Qin, H.; Chen, X.; Ma, J.; Zhou, J.; Chen, L.; Wang, L.; Wang, L. W.; Peng, X. Engineering Auger Recombination in Colloidal Quantum Dots via Dielectric Screening. Nat. Commun. 2019, 10, 1750. https://doi.org/10.1038/s41467-019-09737-2.

(9) Wang, L.; Chen, Z.; Liang, G.; Li, Y.; Lai, R.; Ding, T.; Wu, K. Observation of a Phonon Bottleneck in Copper-Doped Colloidal Quantum Dots. Nat. Commun. 2019, 10, 4532. https://doi.org/10.1038/s41467-019-12558-y.

(10) Tyagi, P.; Cooney, R. R.; Sewall, S. L.; Sagar, D. M.; Saari, J. I.; Kambhampati, P. Controlling Piezoelectric Response in Semiconductor Quantum Dots via Impulsive Charge Localization. Nano Lett. 201010 (8), 3062-3067. https://doi.org/10.1021/nl101605r.

(11) Kelley, A. M.; Dai, Q.; Jiang, Z. J.; Baker, J. A.; Kelley, D. F. Resonance Raman Spectra of Wurtzite and Zincblende CdSe Nanocrystals. Chem. Phys. 2013, 422, 272-276. https://doi.org/10.1016/j.chemphys.2012.09.029.

(12) Li, B.; Brosseau, P. J.; Strandell, D. P.; Mack, T. G.; Kambhampati, P. Photophysical Action Spectra of Emission from Semiconductor Nanocrystals Reveal Violations to the Vavilov Rule Behavior from Hot Carrier Effects. J. Phys. Chem. C 2019123 (8), 5092-5098. https://doi.org/10.1021/acs.jpcc.8b11218.

(13) Mooney, J.; Krause, M. M.; Saari, J. I.; Kambhampati, P. Challenge to the Deep-Trap Model of the Surface in Semiconductor Nanocrystals. Phys. Rev. B - Condens. Matter Mater. Phys. 2013, 87 (8), 081201. https://doi.org/10.1103/PhysRevB.87.081201.

(14) Mack, T. G.; Jethi, L.; Kambhampati, P. Temperature Dependence of Emission Line Widths from Semiconductor Nanocrystals Reveals Vibronic Contributions to Line Broadening Processes. J. Phys. Chem. C 2017, 121 (51), 28537-28545.

https://doi.org/10.1021/acs.jpcc.7b09903. 
(15) Jethi, L.; Mack, T. G.; Kambhampati, P. Extending Semiconductor Nanocrystals from the Quantum Dot Regime to the Molecular Cluster Regime. J. Phys. Chem. C 2017121 (46), 26102-26107. https://doi.org/10.1021/acs.jpcc.7b08439.

(16) Mooney, J.; Kambhampati, P. Get the Basics Right: Jacobian Conversion of Wavelength and Energy Scales for Quantitative Analysis of Emission Spectra. J. Phys. Chem. Lett. 2013, 4 (19), 3316-3318. https://doi.org/10.1021/jz401508t.

(17) Bodunov, E. N.; Danilov, V. V.; Panfutova, A. S.; Simões Gamboa, A. L. Room-Temperature Luminescence Decay of Colloidal Semiconductor Quantum Dots: Nonexponentiality Revisited. Ann. Phys. 2016, 528, 272-277. https://doi.org/10.1002/andp.201500350.

(18) Bodunov, E. N.; Antonov, Y. A.; Simões Gamboa, A. L. On the Origin of Stretched Exponential (Kohlrausch) Relaxation Kinetics in the Room Temperature Luminescence Decay of Colloidal Quantum Dots. J. Chem. Phys. 2017 146, 114102. https://doi.org/10.1063/1.4978396.

(19) Bodunov, E. N.; Simões Gamboa, A. L. Photoluminescence Decay of Colloidal Quantum Dots: Reversible Trapping and the Nature of the Relevant Trap States. J. Phys. Chem. C 2019, 123 (41), 25515-25523. https://doi.org/10.1021/acs.jpcc.9b07619.

(20) Baikie, I. D.; Mackenzie, S.; Estrup, P. J. Z.; Meyer, J. A. Noise and the Kelvin Method. Rev. Sci. Instrum. 1991, 62, 1326. https://doi.org/10.1063/1.1142494.

(21) Szemjonov, A.; Galkowski, K.; Anaya, M.; Andaji-Garmaroudi, Z.; Baikie, T. K.; Mackowski, S.; Baikie, I. D.; Stranks, S. D.; Islam, M. S. Impact of Oxygen on the Electronic Structure of Triple-Cation Halide Perovskites. ACS Mater. Lett. 2019, 1 (5), 506-510. https://doi.org/10.1021/acsmaterialslett.9b00294.

(22) Nonnenmacher, M.; O’Boyle, M. P.; Wickramasinghe, H. K. Kelvin Probe Force Microscopy. Appl. Phys. Lett. 1991, 58 (25), 2921-2923. https://doi.org/10.1063/1.105227.

(23) Sadewasser, S.; Glatzel, T.; Rusu, M.; Jäger-Waldau, A.; Lux-Steiner, M. C. High-Resolution Work Function Imaging of Single Grains of Semiconductor Surfaces. Appl. Phys. Lett. 2002, 80 (16), 2979-2981. https://doi.org/10.1063/1.1471375.

(24) Jaramillo-Quintero, O. A.; Triana, M. A.; Rincon, M. E. Optimization of Charge Transfer and Transport Processes at the CdSe Quantum Dots/TiO2 Nanorod Interface by TiO2 Interlayer Passivation. J. Phys. D. Appl. Phys. 2017, 50, 235305. https://doi.org/10.1088/13616463/aa6e7c. 